\title{
Influence of Adsorption and Capillary Pressure on Phase Equilibria Inside Shale Reservoirs
}

Sandoval, Diego R.; Yan, Wei; Michelsen, Michael L.; Stenby, Erling H.

Published in:

Energy and Fuels

Link to article, DOI:

10.1021/acs.energyfuels.7b03274

Publication date:

2018

Document Version

Peer reviewed version

Link back to DTU Orbit

Citation (APA):

Sandoval, D. R., Yan, W., Michelsen, M. L., \& Stenby, E. H. (2018). Influence of Adsorption and Capillary Pressure on Phase Equilibria Inside Shale Reservoirs. Energy and Fuels, 32(3), 2819-2833.

https://doi.org/10.1021/acs.energyfuels.7b03274

\section{General rights}

Copyright and moral rights for the publications made accessible in the public portal are retained by the authors and/or other copyright owners and it is a condition of accessing publications that users recognise and abide by the legal requirements associated with these rights.

- Users may download and print one copy of any publication from the public portal for the purpose of private study or research.

- You may not further distribute the material or use it for any profit-making activity or commercial gain

- You may freely distribute the URL identifying the publication in the public portal 


\title{
Influence of Adsorption and Capillary Pressure on Phase Equilibria Inside Shale Reservoirs
}

\author{
Diego R. Sandoval, ${ }^{\dagger}$ Wei Yan, ${ }^{* \dagger}{ }^{\dagger}$ Michael L. Michelsen, ${ }^{\dagger}$ and Erling H. Stenby ${ }^{\dagger}$ \\ $\dagger$ Center for Energy Resources Engineering (CERE), Department of Chemistry, Technical \\ University of Denmark, Lyngby 2800 \\ $\ddagger$ Center for Energy Resources Engineering (CERE), Department of Chemical Engineering, \\ Technical University of Denmark, Lyngby 2800 \\ E-mail: weya@kemi.dtu.dk*
}

\begin{abstract}
Due to the small pore sizes and organic content of shale, capillary pressure and adsorption are two effects that should be taken into account in the study of phase equilibrium inside shale. The inclusion of both effects in the phase equilibrium modeling can shed light on how bulk phase composition inside the porous media changes with temperature and pressure, and how the phase equilibrium changes accordingly. In the long run, such a model can be used in reservoir simulation for more complicated analysis. In this study, we present a calculation method that can effectively include adsorption and capillarity. We propose to introduce an excess adsorbed phase and treat the remaining substance inside the pores as a bulk phase (gas, liquid, or both) in order to make the mass balance formulation simpler. The adsorbed phase is modeled by the Multicomponent Langmuir (ML) equation for its simplicity and computational efficiency. A more theoretical adsorption model, the multicomponent potential theory of adsorption (MPTA), is used to determine the parameters of the simpler ML equation. The liquid and gas phases are described by the Peng-Robinson equation of state and
\end{abstract}


the capillary pressure across their interface is taken into account. A flash algorithm by alternately updating the adsorbed phase amount and the fugacities in the bulk phases has been developed. The flash algorithm is used to analyze some representative systems (from binary, ternary to low-GOR and high-GOR model reservoir fluid systems) for the phase equilibrium inside porous media. The results show that adsorption and capillary pressure can significantly change the bulk phase composition and thus its corresponding phase envelope. Since the adsorption varies at different temperature and pressure conditions, the extent of change in the phase envelope is different. In general, a much shrunk phase envelope with a shifted critical point is observed. The heavier components are preferentially adsorbed in the whole pressure and temperature range studied here. At high pressure and low temperature, the selectivity towards heavier components is moderate in comparison to the that at low pressure and high temperature. The adsorption effects are stronger for the gas bulk phase region, leading to bigger changes in the gas phase composition and the shift of the dew point curve. PVT simulations of two model reservoir fluid systems show significant change in the results when capillary pressure and adsorption are included.

\section{Introduction}

Production of oil and gas from shale reservoirs has gained more attention in the past decade due to its increasing economic feasibility and the size of potential sources around the world. The improvement of technologies such as horizontal drilling and hydraulic fracturing has

allowed to economically produce from such type of reservoirs. ${ }^{1}$ Production from shale has been the fastest growing energy sector in the United States, being able to partly substitute electricity production from coal-fired power plants. ${ }^{2}$ A similar trend is expected in countries with huge potential sources of shale such as Canada, China and Argentina. ${ }^{3}$

Shale reservoirs are characterized to have a very heterogeneous rock with noticeable organic and inorganic regions, a wide range of pore size distributions with average pore 
sizes in the nanometer scale, and low porosities. These characteristics pose challenges in the understanding and description of several underlying phenomena critical to shale production, such as flow and transport, rock mechanics, and phase behavior. For instance, abnormal production profiles in tight oil and shale oil reservoirs cannot be explained with the current framework, ${ }^{4}$ suggesting that additional effects on phase behavior may be needed in the current models and simulation tools. Nojabaei et al. ${ }^{5}$ and Kurtoglu et al. ${ }^{6}$ have reported production data from different shale-oil wells of the Bakken field with long-lasting periods of constant GOR at pressures below the expected saturation point. The understanding of phase behavior is crucial in reservoir simulation tools in order to forecast oil production accurately. Two important effects altering the phase behavior in shale reservoirs are high capillary forces and surface interactions.

The capillary pressure effect has been studied theoretically and experimentally for pure components and mixtures. Fisher and Israelachvili ${ }^{7}$ validated the Kelvin equation for pure components at pore radii of $4 \mathrm{~nm}$ for cyclohexane. However, for the multicomponent case, the experimental measurements are more challenging and few data can be found. The recent interests in shale gas production have led to several experimental investigations. ${ }^{8-13}$ Wang et al. ${ }^{8}$ and Alfi et al. ${ }^{9}$ used nano-fluidics to study the vaporization of pure components and mixtures in nano-scale channels. Luo et al. ${ }^{11-13}$ used differential calorimetry to measure the change in the boiling point of mixtures inside nano-scale porous media at atmospheric pressure. Pathak et al. ${ }^{10}$ attempted to detect the bubble point change of $\mathrm{C}_{1}$ - $\mathrm{C}_{10}$ system in well-characterized synthesized mesoporous materials using the classical pressure-volume curve measurement. The findings from these experiments are not always consistent with each other. On the other hand, theoretical and modeling studies have also been done by several authors. Brusilovsky ${ }^{14}$ presented a mathematical simulation for hydrocarbon mixtures under a capillary pressure difference showing differences in the saturation pressure. Shapiro and Stenby ${ }^{15-17}$ formulated the multicomponent Kelvin equation and presented a thermodynamic analysis in which the phase equilibrium conditions for a mixture under capillary pressure are 
established. Sandoval et al. ${ }^{18}$ presented a linear analysis and an algorithm for phase envelope calculations under a capillary pressure difference and observed changes in the whole phase envelope except in the critical point. In the reservoir simulation context, several authors have included the effect of capillary pressure in the phase behavior, reporting deviations in the production profiles. $5,19 ?, 20$

Adsorption of components to the rock can be another important effect on phase behavior. The thickness of adsorption film modifies the capillary radius and enhances the capillary effect. ${ }^{21}$ Moreover, selective adsorption may occur modifying the composition in the bulk space of the pore. Without considering this compositional changes, incorrect predictions on fluid properties may be obtained. For reliable predictions accounting for adsorption effects, experimental adsorption data of different hydrocarbons at different temperatures and pressures is crucial. Several authors have reported adsorption data in shale for methane, ethane, $\mathrm{CO}_{2}$ and their binary mixtures at a wide range of conditions both from experiments and molecular simulations. ${ }^{22-29}$ However, there are no experimental data for simultaneous phase equilibrium and adsorption of single components and their mixtures in shale. Therefore, tools that can give us an insight on the main characteristics of the physical picture are valuable to design experimental setups, and in the long run, understand the phase behavior during the production of shale reservoirs.

In this work we present a novel algorithm for flash calculations inside shale reservoirs, where both the capillary pressure and adsorption effects are taken into account. Extended from the conventional two-phase flash, the new algorithm can describe the simultaneous equilibrium between the bulk gas and liquid phases and the adsorbed phase. It accounts for the capillary pressure between two bulk phases and the overall composition change of the bulk phases due to selective adsorption of components to the wall, as detailed in the Methodology section. In the Solution Procedure sections, we attempt to increase the robustness and efficiency of the algorithm so that it can be used not only for analyzing the capillarity and adsorption influence of static scenarios over a wide temperature and pressure range as in this 
work, but also for future dynamic analysis through integration into compositional simulation. In the Results section, the algorithm is used to analyze four model systems representative for shale reservoir fluids. The changes in phase equilibrium and their underlying reasons are discussed.

\section{Methodology}

Isothermal flash calculations are perhaps the most important phase equilibrium calculations in practical applications. They are at the core of compositional reservoir simulations and many process engineering tools to determine the equilibrium phase composition and properties at specified pressure and temperature. Incorporation of the adsorption effects and capillary pressure in isothermal flash calculations is essential to the analysis of phase equilibrium inside shale reservoirs, and inside confined systems in general.

Capillarity and adsorption of components to the rock introduce additional constraints to the conventional isothermal two-phase flash problem. The former introduces a difference of pressures between the liquid and gas phases, and the latter introduces a new phase that changes the distribution of components among the phases. An example of a confined system under capillarity and adsorption is depicted by Figure 1. It shows a fluid inside a pore with specific surface area $A$ and void volume $V$. A total of three phases are present in the system: a liquid phase, a gas phase, and an adsorbed phase. A capillary pressure difference $P_{c}$ is accounted for across the interface of the liquid and gas phases. Although a cylindrical geometry is shown, the proposed method can be applied to any system with a known void volume and specific surface area.

The goal of the flash procedure is to determine the phase fractions and compositions of the adsorbed phase, liquid phase, and gas phase in the system at a specified pressure $P$, temperature $T$, and overall composition $\mathbf{z}^{\mathbf{f}}$. When the system is at equilibrium, we can write 

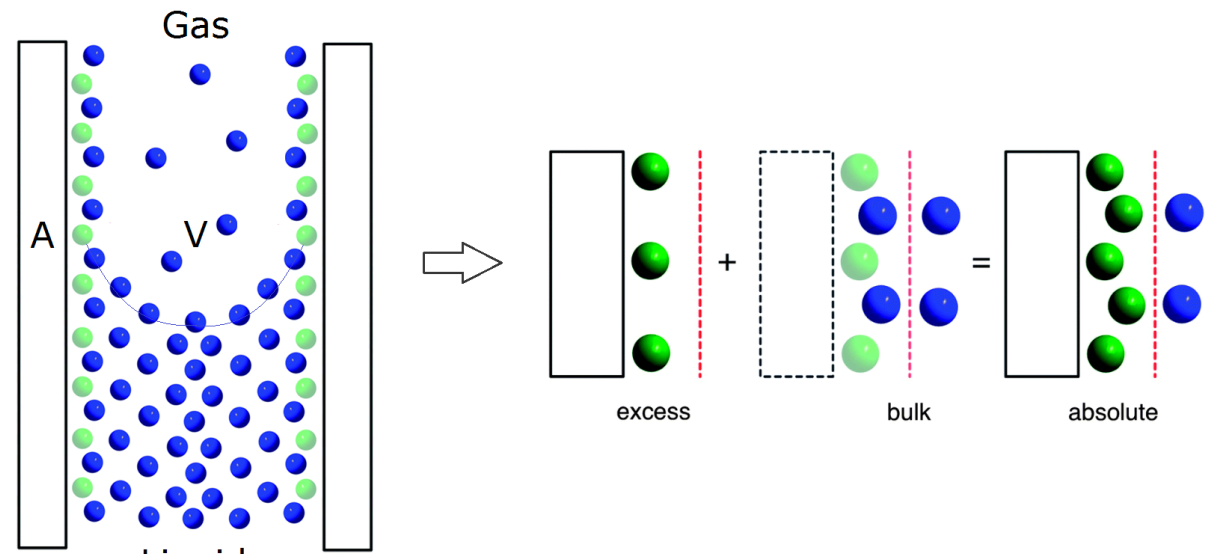

Liquid

Figure 1: Blue spheres correspond to the bulk phases (gas and liquid), and green spheres correspond to the excess adsorbed phase. The first blue/green layer next to the wall corresponds to the absolute adsorbed layer. The liquid/gas curved interface represent the capillary pressure difference. Figure adapted from Mason et al. ${ }^{30}$

that the fugacity of each component in the liquid, gas, and adsorbed phase is the same.

$$
f_{i}^{g}=f_{i}^{l}=f_{i}^{a}
$$

where $f_{i}^{\alpha}$ is the fugacity of component $i$ in phase $\alpha$. Throughout the entire work the superscripts $g, l$ and $a$ are used for the gas, liquid and adsorbed phases respectively. The system is subject to mass balance constraints:

$$
z_{i}^{f}=\theta^{g} y_{i}+\theta^{l} x_{i}+\theta^{a} w_{i}
$$

where $z_{i}^{f}$ is the normalized feed composition, $y_{i}$ and $\theta^{g}$ are the molar compositions and molar phase fraction in the vapor phase; $x_{i}$ and $\theta^{l}$ in the liquid phase; and $w_{i}$ and $\theta^{a}$ in the excess adsorbed phase. The summation of the phase fractions must sum to unity

$$
\theta^{l}+\theta^{g}+\theta^{a}=1
$$


and the molar compositions in each phase must also sum to unity.

$$
\sum_{i} y_{i}=\sum_{i} x_{i}=\sum_{i} w_{i}=1
$$

It is advantageous to use the excess adsorbed phase fraction instead of the absolute one for the mass balance. In Figure 1 we can notice that the space inside the cylinder is filled by the bulk phases (blue spheres) and the absolute adsorbed phase (first layer next to the wall). Furthermore, to delimit the space that corresponds to the adsorbed phase and the bulk phases, it is necessary to introduce an assumption on the thickness of the adsorbed layer. However, this issue can be avoided by utilizing the excess adsorbed phase fraction (green spheres in Figure 1) instead of the absolute adsorbed phase fraction. The excess adsorbed phase occupies empty spaces that the bulk phase would not occupy in the absence of an adsorbed phase. As a consequence, the bulk phase amounts can be calculated using the total volume $\mathrm{V}$, and the excess amounts using the surface area A. The details are presented in the solution procedure section.

In this work, the thickness of the adsorption film is neglected in the contribution of the capillary pressure for practical purposes. The error introduced by neglecting the adsorption thickness for radii greater than $5 \mathrm{~nm}$ is relatively small far away from the critical point and almost negligible close to the critical point due to low interfacial tension values as shown in our previous work. ${ }^{31}$ Hence, the pore radius and effective capillary radius are treated the same here. The Young-Laplace equation inside a cylinder is employed to describe the capillary pressure difference

$$
P_{c}=P^{g}-P^{l}=\frac{2 \sigma}{r_{c}}
$$

where $P_{c}$ is the capillary pressure, $P^{g}$ the pressure in the gas phase, $P^{l}$ the pressure in the liquid phase (wetting phase), and $\sigma$ the interfacial tension, which for this work is calculated 
using the Sugden and Macleod model, commonly known as the parachor model

$$
\sigma=\left[\sum_{i} \chi_{i}\left(x_{i} \rho^{l}-y_{i} \rho^{g}\right)\right]^{4}
$$

where $\chi_{i}$ are the parachor constants, $\rho^{l}$ and $\rho^{g}$ are the molar densities in $\left(\mathrm{mol} / \mathrm{cm}^{3}\right)$, and $\sigma$ is the interfacial tension in $\left(d y n e / \mathrm{cm}^{3}\right)$. Furthermore, it is recommended to use a capillary pressure model according to its geometry. For instance, for heterogeneous porous media, a function that accounts for the pore size distribution, such as Leverett J-function ${ }^{32}$ must be employed.

The liquid and gas phases are modeled using the Peng-Robinson EoS, although other EoS can be selected. For the scope of this study, the adsorbed phase exists as long as there is a bulk phase present. The adsorbed amount is often modeled as a function of the fugacity $\left(\mathbf{f}^{\mathbf{b}}\right)$ of one of the bulk phases in the system. We can therefore write:

$$
n_{a d s}=\Psi\left(\mathbf{f}^{\mathbf{b}}\right)
$$

where $n^{a d s}$ is the adsorbed amount, which can be the excess $n^{e x c}$ or absolute adsorbed amount $n^{a b s}$ depending on the model, $\Psi$ represents the model used for the adsorbed phase, and $f^{b}$ can be either the gas or liquid fugacity if both phases are present in the system. The Multicomponent Langmuir (ML) is used here, but the described framework can be used with any adsorption model. The ML can be written as follows:

$$
n_{i}^{a b s}=n_{i}^{\max }(T) \frac{b_{i}(T) f_{i}}{1+\sum_{j=1}^{N c} b_{j}(T) f_{j}}
$$

where $n_{i}^{a b s}$ is the absolute adsorbed amount for component $i, n_{i}^{\max }$ is the maximum adsorption capacity, and $b_{i}$ is the adsorption equilibrium constant. The Langmuir model has been initially developed to calculate the absolute adsorbed amount. At low pressures, the absolute 
and excess amounts are almost identical but this is not the case at high pressures where the difference is too large to ignore. Since it is of our interest to estimate the excess adsorption at high pressures, modifications to the ML must be done as follows:

$$
n_{i}^{a b s}=n_{i}^{\max }(T) \frac{b_{i}(T) f_{i}}{1+\sum_{j=1}^{N c} b_{j}(T) f_{j}}-\rho^{b} V_{a d s}
$$

where $\rho^{b}$ is the density of the bulk phase and $V_{a d s}$ is the volume of the adsorbed phase, which in many cases can be estimated by the micro-pore volume of the adsorbent or obtained as a fitting parameter during the regression of the adsorption data. In a more realistic scenario, $V_{a d s}$ and surface area $A$ will change due to the swelling or shrinkage of the sample. The swelling/shrinkage may depend on the pressure, temperature and loading of hydrocarbons/water. ${ }^{33-35}$ For practical purposes of this work, we have assumed for the tested examples that swelling and shrinkage does not occur. Nevertheless, the generality of the framework allows to embed this dependency into the adsorption model if required.

\section{Solution Procedure}

In principle, the system can be solved with a nested robust isothermal flash updating the adsorbed amounts in an outer loop. However, we found that it is more convenient and efficient to couple the adsorption calculations with the bulk equilibrium calculations. In

other words, the bulk phase fugacities, the capillary pressure, and the distribution of the phases are calculated at each step.

The developed algorithm follows a similar methodology used for the two-phase flash algorithm by Michelsen ${ }^{36}$ but several modifications to account for the capillary pressure difference and the composition changes in the bulk phase due to adsorption are employed. At early iterations, it is based on a direct substitution procedure of the equilibrium factors solving the Rachford-Rice equation in a nested loop, while updating the capillary pressure 
and adsorbed amounts in an external loop. Acceleration using the dominant eigenvalue of the equilibrium factors and changes in the bulk composition are performed whenever the eigenvalues responsible for the slow convergence are identified and isolated. The procedure is then switched at later iterations to a quadratic update in the vapor flows while maintaining the direct substitution update in the capillary pressure and adsorbed amounts.

\section{Direct Substitution}

As a starting point, the pressure in the liquid phase and the gas phase is assumed equal, the adsorbed phase fraction is set to zero, and the Wilson's correlation is used to obtain an initial estimate of the equilibrium factors

$$
\ln K_{i}^{\text {Wilson }}=\ln \frac{P_{c_{i}}}{P}+5.737\left(1+w_{i}\right)\left(1-\frac{T_{c_{i}}}{T}\right)
$$

where $T_{c_{i}}, P_{c_{i}}$ and $w_{i}$ are the critical temperature, critical pressure and acentric factor respectively. The calculation is followed by solving the Rachford-Rice equation iteratively with the corresponding update of the equilibrium factors, capillary pressure and total bulk composition changes due to adsorption at each step. The Rachford-Rice can be written as follows:

$$
g(\beta)=\sum_{i} z_{i}^{b} \frac{K_{i}-1}{1-\beta+\beta K_{i}}=0
$$

where $\mathbf{z}^{\mathbf{b}}$ is the total composition of the bulk phase (i.e. liquid and gas together), $K_{i}$ are the equilibrium factors between the gas and the liquid, and $\beta$ is the relative vapor fraction in the bulk phase without considering the adsorbed phase. The relative vapor fraction is not equivalent to the absolute fraction $\theta^{g}$ and both are related through the following equation:

$$
\beta=\frac{\theta^{g}}{\theta^{g}+\theta^{l}}
$$


Updates of the capillary pressure $P_{c}$, equilibrium factors $K_{i}$, and bulk composition $\mathbf{z}_{\mathbf{b}}$ at each solution of (Eq. 11) are performed in the respective order. The capillary pressure is computed using (Eq. 5) and the update of the phase pressures is made by

$$
P^{g}=P^{l}+P_{c} \quad \text { or } \quad P^{l}=P^{g}-P_{c}
$$

where $P^{g}$ is updated if the pressure of the liquid is the input pressure, and $P^{l}$ if the pressure of the gas is the input. It can be noted that at high values of $P_{c}$ and small values of $P_{g}$ the update in the liquid pressure can be negative. Therefore, it is always recommended to use the product of the pressure and fugacity coefficient (i.e. $F_{i}^{\alpha}=P^{\alpha} \varphi_{i}^{\alpha}$ ), instead of the fugacity coefficients $\varphi_{i}^{\alpha}$ alone to avoid undefined values during intermediate calculations. ${ }^{18}$ After updating the capillary pressure, the $K_{i}$ values are calculated as follows:

$$
K_{i}=\frac{F_{i}^{l}\left(T, P^{l}, \mathbf{x}\right)}{F_{i}^{g}\left(T, P^{g}, \mathbf{y}\right)}
$$

Finally, the last variable to be updated is the total bulk composition $\mathbf{z}_{\mathbf{b}}$. The total composition in the bulk phase will change at each step since some components will be adsorbed to the wall. By subtracting the components of the adsorbed phase from the overall feed composition we get:

$$
z_{i}^{b}=\frac{z_{i}^{f}-\theta^{a} w_{i}}{1-\theta^{a}}
$$

The composition of the excess adsorbed phase $\mathbf{w}$ is obtained from (Eq. 7) using the liquid fugacity, gas fugacity, or an average of both. This is allowed during intermediate calculations since at the solution both the liquid and gas phases will have the same fugacity. The absolute mole fractions $\mathbf{x}^{\text {abs }}$ can be obtained with the ML model

$$
x_{i}^{a b s}=\frac{n_{i}^{a b s}}{\sum_{j} n_{j}^{a b s}}
$$

However, to obtain the composition in the excess adsorbed phase we have to make the 
correction for the excess amounts.

$$
n_{i}^{e x c}=n_{i}^{a b s}-z_{i}^{b} \rho^{b} V^{a d s} \quad \rightarrow \quad w_{i}=\frac{n_{i}^{e x c}}{\sum_{j} n_{j}^{e x c}}
$$

where $V^{a d s}$ is obtained by fitting the excess adsorption data of pure components, and $\rho^{b}$ is the overall density of the bulk phase

$$
\rho^{b}=\frac{1}{\beta V_{m}^{g}+(1-\beta) V_{m}^{l}}
$$

where $V_{m}^{l}$ and $V_{m}^{g}$ are the molar volumes of the liquid and gas phase respectively.

The remaining excess adsorbed phase fraction $\theta^{a}$ in (Eq. 15), can be obtained using the phase molar densities (or volumes) and the geometry of the system. Since our calculation is done at constant pressure, the volume is allowed to change, but the ratio of the surface area to the void volume remains constant. We can rewrite the surface area, and the volume of the system as follows:

$$
A=\frac{n^{e x c}}{\Gamma^{e x c}} \quad, \quad V=n^{b} \bar{V}_{m}
$$

where $\Gamma^{e x c}$ is the surface excess (excess adsorbed moles per unit area); $\bar{V}_{m}$ is the molar volume of the bulk, $n^{b}$ are the moles in the bulk phase (i.e. $n^{b}=n^{l}+n^{g}$ ), and $n^{e x c}$ the moles in the excess adsorbed phase. The molar volume of bulk refers to the average molar volume of the liquid and gas phases

$$
\bar{V}_{m}=\beta V_{m}^{g}+(1-\beta) V_{m}^{l}
$$

where $\beta$ is the relative vapor fraction in (Eq. 12). Using the fact that the geometric factor $G_{f}=A / V$ is constant for a specific porous media, (Eq. 19) can be used to express the geometric factor as follows:

$$
G_{f}=\frac{n^{e x c} / \Gamma^{e x c}}{n^{b} \bar{V}_{m}}=\frac{\theta^{a}}{\left(1-\theta^{a}\right) \Gamma^{e x c} \bar{V}_{m}}
$$


Solving for the excess adsorbed fraction we obtain

$$
\theta^{a}=\frac{G_{f} \bar{V}_{m} \Gamma^{e x c}}{1+G_{f} \bar{V}_{m} \Gamma^{e x c}}
$$

For a cylinder, the geometric factor is $\frac{2}{r_{c}}$. With (Eq. 22), the update of $\mathbf{z}_{\mathbf{b}}$ in (Eq. 15) is complete.

It is worthwhile to notice that the excess adsorbed amount $n_{i}^{e x c}$ of some components in the excess adsorbed phase may be negative. This happens in cases where there is a higher concentration of one component in the bulk phase than in the excess adsorbed phase

$$
n_{i}^{e x c}=n_{i}^{a d s}-z_{i}^{b} \rho^{b} V^{a d s}<0
$$

This is not unusual when the system consists of a liquid phase and an adsorbed phase. This shows that the excess adsorbed phase is merely a hypothetical concept instead of a separate physical entity. The mole fractions in this hypothetical phase fulfill the mass balance equations and are not directly used to calculate other thermodynamic properties. Therefore, it is not a problem for $w_{i}$ to be negative during the calculations and at the solution.

In summary, an iterative procedure solving the Rachford-Rice equation using direct substitution in the equilibrium factors $K_{i}$ (Eq. 14), capillary pressure $P_{c}$ (Eq. 13), and bulk concentration $\mathbf{z}_{\mathbf{b}}$ (Eq. 15) can be performed until reaching a desired tolerance or number of iterations. The convergence rate can be improved by using an extrapolation method. In this work the Dominant Eigenvalue Method (DEM) suggested by Orbach and Crowe ${ }^{37}$ is employed to accelerate convergence on the equilibrium factors and bulk compositions.

\section{Quadratic Update on Vapor Flows}

A further increase in the convergence rate can be achieved using a second order method in the phase split of the bulk phases while maintaining a direct substitution update in $P_{c}$ and $\mathbf{z}_{\mathbf{b}}$. This procedure is especially useful when the bulk phases are close to the critical point, 
where a large number of successive substitution iterations are required.

The equilibrium equations of the gas and liquid phases can be written as a function of the vapor flows as independent variables

$$
g_{i}(\mathbf{v})=\ln f_{i}^{g}-\ln f_{i}^{l}=0
$$

The Jacobian matrix for the system in (Eq. 24) can be obtained as follows

$$
J_{i j}=\frac{1}{\theta(1-\theta)}\left(\frac{z_{i}^{b}}{x_{i} y_{i}} \delta_{i j}-1+(1-\theta) \Phi_{i j}^{g}+\theta \Phi_{i j}^{l}\right)
$$

where

$$
\Phi_{i j}^{\alpha}=n_{T}\left(\frac{\partial \ln \varphi_{i}}{\partial n_{j}}\right)_{T, P^{\alpha}} \quad, \quad n_{T}=\sum_{k} n_{k}
$$

For details in the derivation, the reader is referred to Michelsen and Mollerup. ${ }^{36}$ The Newton update in the vapor flows can be obtained by solving the following system

$$
\mathbf{J} \Delta \mathbf{v}+\mathbf{g}=\mathbf{0}
$$

It is important to use the second order method with high-quality initial estimates in order to have local convergence. It is recommended to perform some cycles of successive substitution followed by acceleration before switching to the quadratic update in the vapor flows.

In addition to the phase split calculation procedure, stability analysis in the bulk phase is performed when necessary. If the bulk phase converges to a single phase at the solution, stability analysis including capillary pressure, suggested by Sherafati et al., ${ }^{38}$ is performed to check whether the obtained solution is stable. If unstable, the new composition is used and the algorithm is restarted.

In summary, the solution procedure is as follows: 
i Initialize with Wilson's equilibrium constants for the bulk phases.

ii Perform accelerated successive substitution using (Eq. 11), (Eq. 13), (Eq. 14), and (Eq. 15).

iii Continue with the second order method for the bulk phase vapor flows while updating by means of successive substitution the capillary pressure and bulk compositions in the outer loop. (Eq. 27)

iv If one of the bulk phases disappears at any point during steps ii) or iii) continue with the phase split of single bulk phase and adsorbed phase. Check the final solution with stability analysis. If stable terminate, otherwise return to step ii.

\section{Results}

The suggested procedure given in the previous sections is able to handle the tested systems summarized in Table 1. The Peng-Robinson EoS was used to model the bulk phase and the ML model for the adsorbed phase. For the adsorbed phase in the examples presented here, we tried to establish our modeling based on experimental adsorption data. A set of adsorption data for methane and ethane recently measured by Wang et al. ${ }^{26}$ was used as a starting point and then extended to different temperatures and heavier n-alkanes.

Various adsorption models can be used to model multicomponent adsorption on shale, for instance: the ML, the Ideal Adsorbed solution Theory (IAST), ${ }^{39}$ and the Multicomponent Potential Theory of Adsorption (MPTA). ${ }^{40}$ All of them show comparable results for adsorption in shale at high pressures. ${ }^{31}$ The most theoretical sound model among them is the MPTA and the simpler to use for calculation purposes is the ML. We here suggest regressing MPTA parameters first based on the available adsorption data, and then use the model to extrapolate to other temperatures and heavier n-alkanes. The artificial adsorption data generated by MPTA can then be used to obtain the temperature dependent model param- 
eters of the Langmuir isotherms. This procedure provides a sound basis for the adsorption calculation part in our model analysis. The details are presented in the supporting information. For the sub-critical region Langmuir isotherms might not be the optimal choice to fit the pure component adsorption data and more complex isotherm models can be applied if required. ${ }^{41}$

The component EoS parameters and parachor constants are presented in Table 2, and the adsorption parameters used for the ML model are presented in Table 3. Furthermore, a cylindrical geometry with a capillary radius $\left(r_{c}\right)$ of $10 \mathrm{~nm}$ was assumed for all the calculations. It is important to notice that the framework formulated here does not have any limitations in the geometry assumed for the system. In the current examples, we have simplified the geometry of the adsorbent to a cylinder in order to use a simple model for the capillary pressure. However, it is also possible to use a more complex capillary pressure model accounting for the pore size distribution. For instance, the Leverett function can be employed to describe the capillary pressure as a function of saturation. The major complication of using a liquid saturation dependent capillary pressure model is that the implicitness of the flash problem increases substantially. Nevertheless, the general calculation framework presented here can still be used. ${ }^{42}$ It is expected that inclusion of the pore size distribution will influence the extent of the change but the major qualitative features will be similar.

Table 1: Molar composition of the tested systems.

\begin{tabular}{lcccc}
\hline Alkane & Binary & Ternary & Low GOR & High GOR \\
\hline $\mathrm{C}_{1}$ & 0.50 & 0.42 & 0.7000 & 0.8997 \\
$\mathrm{C}_{2}$ & 0.50 & - & - & - \\
$\mathrm{C}_{4}$ & - & 0.33 & 0.1200 & 0.0300 \\
$\mathrm{C}_{8}$ & - & - & 0.0700 & 0.0297 \\
$\mathrm{C}_{10}$ & - & 0.25 & - & - \\
$\mathrm{C}_{12}$ & - & - & 0.0599 & 0.0232 \\
$\mathrm{C}_{16}$ & - & - & 0.0501 & 0.0174 \\
\hline
\end{tabular}

The algorithm was applied in a fine grid of $500 \times 500$ for each system and the plots are shown from Figure 4 to Figure 14. The average number of iterations to reach convergence 
Table 2: Peng-Robinson EoS component parameters and parachor constants. Parachor constants taken from Schechter and Guo ${ }^{43}$

\begin{tabular}{lccrrr}
\hline Alkane & $T_{\text {crit }}$ & $P_{\text {crit }}$ & $\omega$ & Parachor & $k_{i j}\left(\mathrm{c}_{1} / \mathrm{c}_{n}\right)$ \\
\hline $\mathrm{C}_{1}$ & 190.6 & 45.99 & 0.0115 & 74.05 & - \\
$\mathrm{C}_{2}$ & 305.3 & 48.72 & 0.0995 & 112.91 & -0.0026 \\
$\mathrm{C}_{4}$ & 425.1 & 37.96 & 0.2002 & 193.90 & 0.0168 \\
$\mathrm{C}_{8}$ & 568.7 & 24.90 & 0.3996 & 359.33 & 0.0451 \\
$\mathrm{C}_{10}$ & 617.7 & 21.10 & 0.4923 & 440.69 & 0.0422 \\
$\mathrm{C}_{12}$ & 658.0 & 18.20 & 0.5764 & 522.26 & 0.0500 \\
$\mathrm{C}_{16}$ & 723.0 & 14.00 & 0.7174 & 688.50 & 0.0561 \\
\hline
\end{tabular}

Table 3: Correlation constants for temperature dependent Langmuir parameters.

\begin{tabular}{|c|c|c|c|c|c|}
\hline Alkane & $\frac{E_{a}}{R}(K)$ & $\ln A *$ & $\mathrm{~m}\left(\frac{m m o l}{g \cdot K}\right)$ & $\mathrm{c}\left(\frac{m m o l}{g}\right)$ & $* *$ \\
\hline $\mathrm{C}_{1}$ & -1115.9 & -7.48 & $-2.66 \cdot 10^{-4}$ & 0.3985 & \\
\hline $\mathrm{C}_{2}$ & -1947.9 & -8.13 & $-2.13 \cdot 10^{-4}$ & 0.2931 & \\
\hline $\mathrm{C}_{4}$ & -2758.5 & -7.91 & $-1.09 \cdot 10^{-4}$ & 0.1769 & \\
\hline $\mathrm{C}_{8}$ & -3989.1 & -6.44 & $-3.32 \cdot 10^{-5}$ & 0.0818 & \\
\hline $\mathrm{C}_{10}$ & -4502.1 & -6.11 & $-2.24 \cdot 10^{-5}$ & 0.0637 & \\
\hline $\mathrm{C}_{12}$ & -5871.2 & -8.00 & $-1.14 \cdot 10^{-5}$ & 0.0496 & \\
\hline $\mathrm{C}_{16}$ & -6189.2 & -5.68 & $-5.41 \cdot 10^{-6}$ & 0.0341 & \\
\hline
\end{tabular}

are from 9 to 15 depending on the system. The algorithm shows to be robust and linearly convergent. The main limitations on the convergence rate are the linear updates in $P_{c}$ and $\mathbf{z}_{\mathbf{b}}$, especially the latter.

Two examples of convergence are given in Figure 2 and Figure 3. An attempt of DEM acceleration is performed after every four steps of successive substitution. Figure 2 shows the convergence of a point in the bulk liquid region. For the $\mathrm{C}_{1}-\mathrm{C}_{2}$, after two interations, the gas phase disappears leaving only a liquid phase and an adsorbed phase in the system. Convergence is reached after the seventh iteration and the solution is checked using stability analysis considering capillary pressure. ${ }^{38}$ Similar behavior is observed for the Low-GOR mixture with a slower convergence rate, and less successful acceleration attempts. Figure 3 depicts a more difficult case of convergence behavior close to the bubble point for the $\mathrm{C}_{1}-\mathrm{C}_{2}$ mixture; after four iterations the gas phase disappears and convergence of the liquid-adsorbed 
system is reached after the ninth iteration. Subsequently, stability analysis considering capillary pressure is performed revealing instability of the liquid bulk phase. The gas phase is reintroduced and iteration is continued by (Eq. 27) and accelerated linear updates in the $\mathbf{z}_{\mathbf{b}}$. Finally, convergence is reached around the 20 th iteration. For the Low-GOR mixture, a point inside the two phase region away from the bubble point is selected. The slope of convergence is improved around the 7th iteration where the update of (Eq. 27) is used. Similarly, convergence is reached around the 20th iteration.
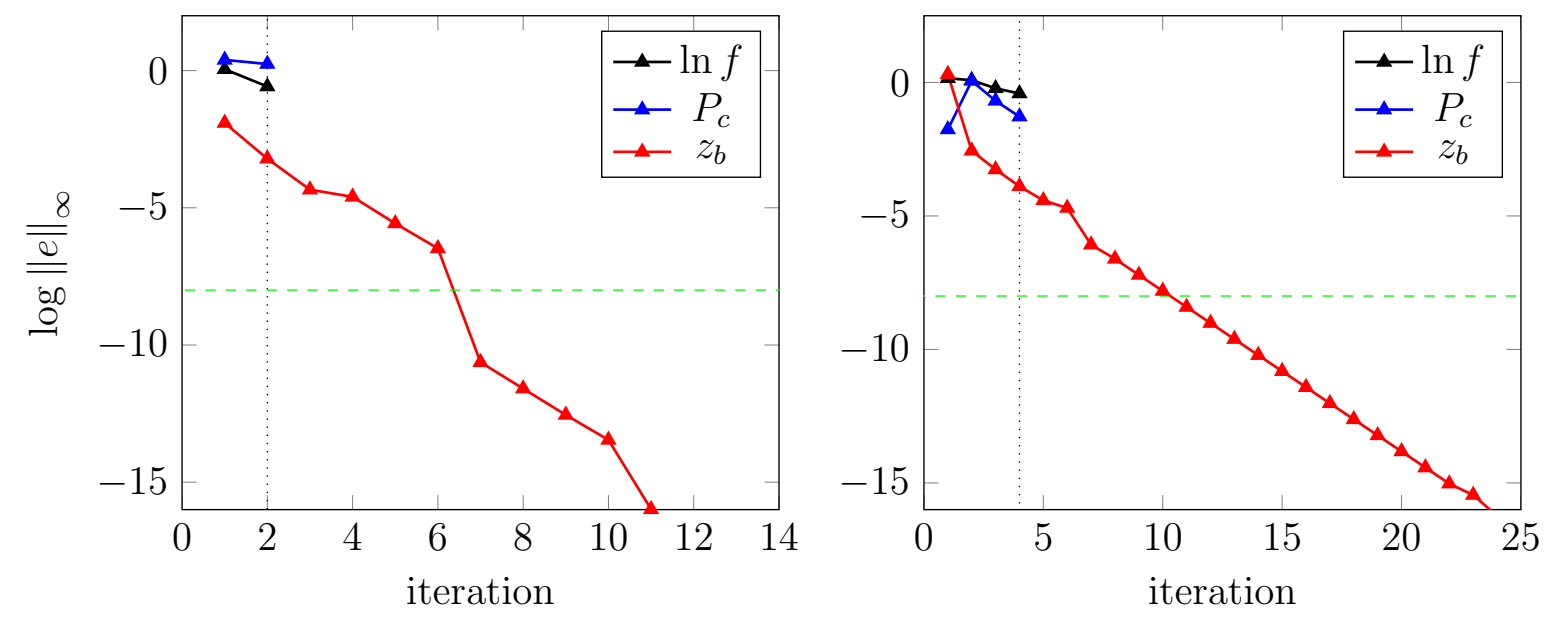

Figure 2: Convergence for the liquid-adsorbed phase region. [Left $] \mathrm{C}_{1}-\mathrm{C}_{2}$ at (240 K, 70 bar). [Right $]$ Low-GOR at $(400 \mathrm{~K}, 400 \mathrm{bar})$. Acceleration attempt in $\mathbf{z}^{\mathbf{b}}$ after each 4 steps. Tolerance limit (- -); Vanishing of the gas phase $(\cdots)$.

\section{Binary System}

The first system tested is an equimolar binary mixture of methane and ethane. The mixture of these two components is of great importance in shale gas reservoirs. The temperatures of two-phase coexistence are extremely low in comparison with the real reservoir conditions. Nevertheless, an example of a binary mixture provides a simple and useful analysis of the main characteristics of the bulk-adsorbed phase splitting. Figure 4 shows the main differences of the phase envelope with and without capillary pressure and adsorption. The phase envelope shows to be considerably smaller and shifted. As shown in our previous work, ${ }^{18}$ 

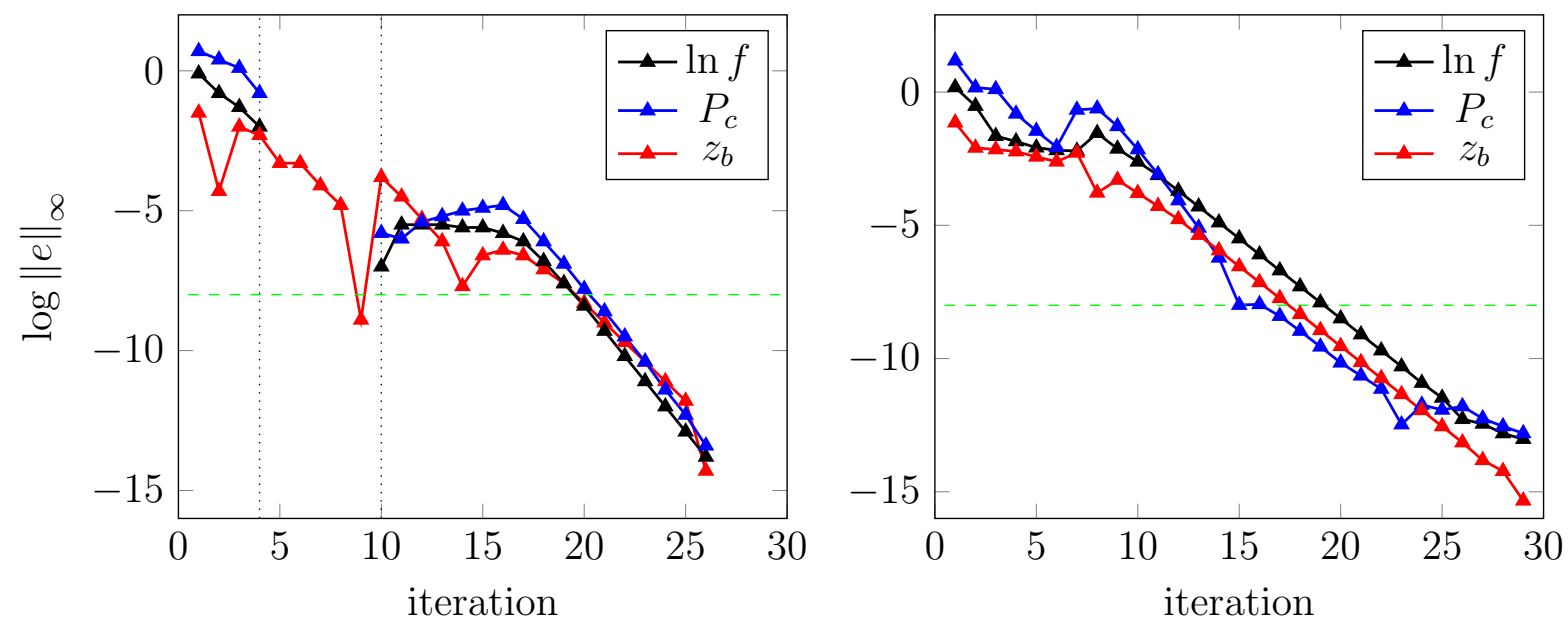

Figure 3: Convergence for the liquid-gas-adsorbed phase region. [Left $] \mathrm{C}_{1}-\mathrm{C}_{2}$ at (240 K, 53.75 bar). [Right] Low-GOR at $\left(400 \mathrm{~K}, 50\right.$ bar). Acceleration attempt in $\mathbf{z}^{\mathbf{b}}$ after each 4 steps. Tolerance limit (- -); Vanishing/appearance of the gas phase $(\cdots)$.

the capillary pressure has an effect on the whole phase envelope except in the critical point. The bubble point pressures and lower dew point pressures are decreased, and the upper dew point pressures increased. The effect of the adsorbed phase is less intuitive. The differences in the phase envelope are due to compositional changes in the bulk phase due to selective adsorption of components to the wall. In other words, each point in the phase diagram has the same overall feed composition $\mathbf{z}_{\mathbf{f}}$, but the bulk composition $\mathbf{z}_{\mathbf{b}}$ is different (see Eq.2).

Figure 5 shows the change of $\mathrm{C}_{2}$ mole fraction in the bulk phase with respect to the feed phase. It is possible to observe that in the liquid region at low temperatures, the composition of the bulk phase changes very little and the change in the phase envelope in this region is mainly caused by capillary effects. In contrast, at very low pressures in the gas region, the composition change in $\mathrm{C}_{2}$ is more dramatic $(\approx-0.4)$ and $\mathrm{C}_{1}$ mole fraction in the bulk phase can get as high as $90 \%$ creating a significantly lighter bulk phase. Moreover, one can expect excess adsorbed phase fractions up to 0.8 (Figure 6), due to big differences in the bulk phase and adsorbed phase molar densities.

Figure 6 shows the excess adsorbed phase fraction of the system plotted against the pressure of the liquid phase $\left(P_{l}\right)$, and the pressure of the gas phase $\left(P_{g}\right)$. The aim of these 
plots is to show more clearly the bubble point and the dew point boundaries plotted against their respective feed pressures. $\mathrm{P}_{l}$ for the bubble point branch, and $\mathrm{P}_{g}$ for the dew point branch. For the bubble point boundary at low temperatures, the excess adsorbed phase fraction and the change in the bulk composition is close to zero, meaning that the bulk phase and the absolute adsorbed phase are almost identical. If we decrease the pressure at a constant temperature, when the bubble point pressure is crossed, rapid changes in the composition and phase fractions occur as shown in Figure 5 and Figure 6. When the gas phase appears in the system, $\mathrm{C}_{1}$ is released from the adsorbed phase to the bulk phase more rapidly than $\mathrm{C}_{2}$ creating a lighter phase in the bulk space. Therefore, the two-phase region shrinks and the dew point is encountered at higher pressures as seen in Figure 4.

Other interesting findings are encountered close to the critical point. We here use the classical definition for the critical point of the bulk gas and liquid phases, i.e. the temperature and pressure where the differences between the coexisting gas and liquid phases disappear. Moreover, we assume here that adsorption holds close to the critical point and its thermodynamic properties are obtained through the selected adsorption model, and only depend on the bulk fugacity as an input. For extremely small pores where density fluctuations around the critical point are suppressed, it would be better to adopt a more sophisticated thermodynamic description. An ideal model should have sufficient theoretical basis and also be accurate enough for high pressure mixture critical point prediction. Here, the marked critical points should be understood as the ones predicted completely under the classical thermodynamic framework. In Figure 5 and Figure 6, if we increase the temperature along the bubble point boundary towards the critical point, we can observe that the excess adsorbed phase fraction increases and so does the composition of $\mathrm{C}_{1}$ in the bulk phase. Due to the selective adsorption towards $\mathrm{C}_{2}$ and the resulting change in the bulk phase composition, the critical point of the bulk phase shifts.

In summary, we observe changes in all the PT phase envelope due to capillary pressure and adsorption. The effect of the capillary pressure near the bubble point is more pronounced 
than the compositional changes due to adsorption. Furthermore, selective adsorption towards $\mathrm{C}_{2}$ is observed everywhere. It is moderate at high pressures and low temperatures, but increases as the pressure is lowered.
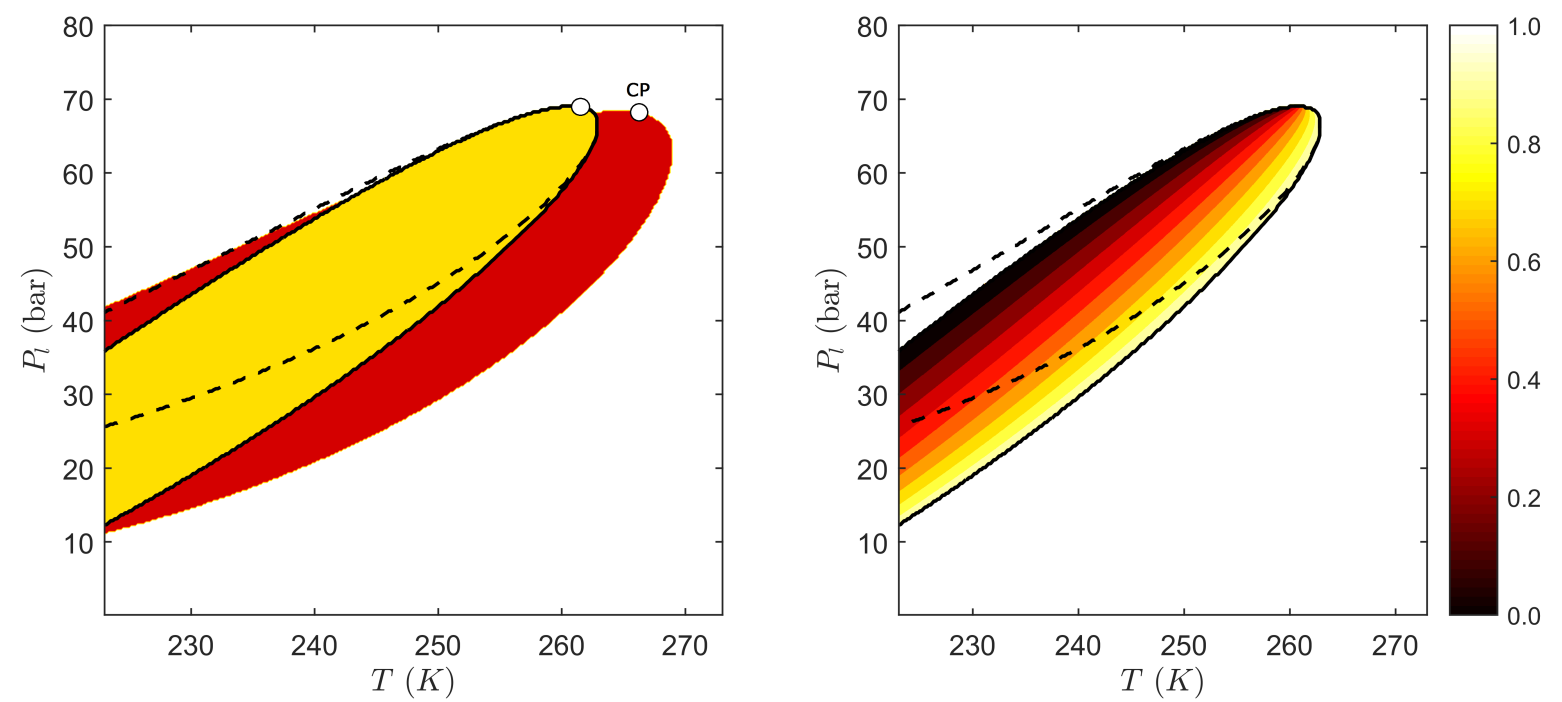

Figure 4: $\mathrm{C}_{1}-\mathrm{C}_{2}$ binary system. [Left] Normal phase envelope in red; with capillary pressure and adsorption in yellow. [Right] Phase envelope with relative vapor fraction $\beta$. The dashed lines (- - ) represent the pressure in the gas phase.
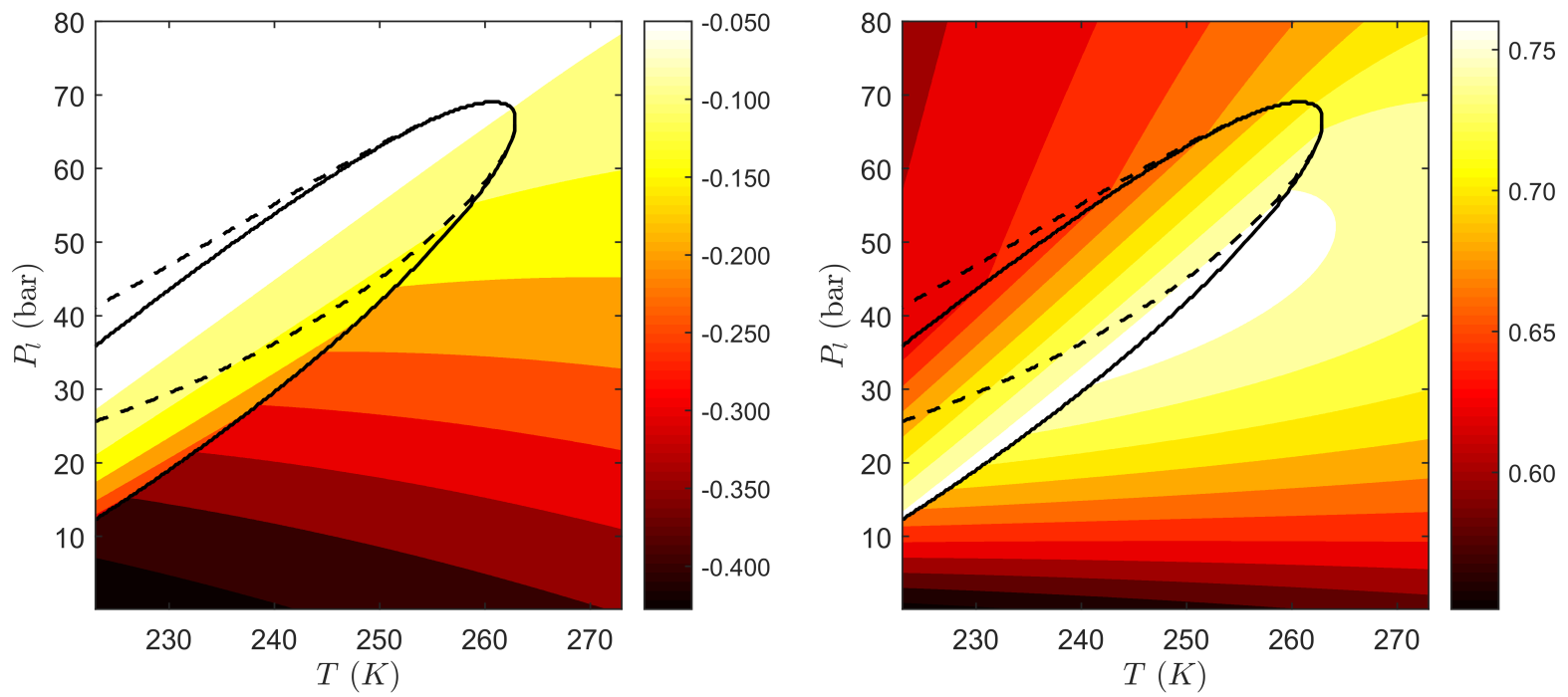

Figure 5: $\mathrm{C}_{1}-\mathrm{C}_{2}$ binary system. [Left] Change of the $\mathrm{C}_{2}$ mole fraction in bulk phase $\mathbf{z}_{\mathbf{b}}$. [Right $] \mathrm{C}_{2}$ mole fraction in the absolute adsorbed phase $\mathrm{x}^{\text {abs }}$. 

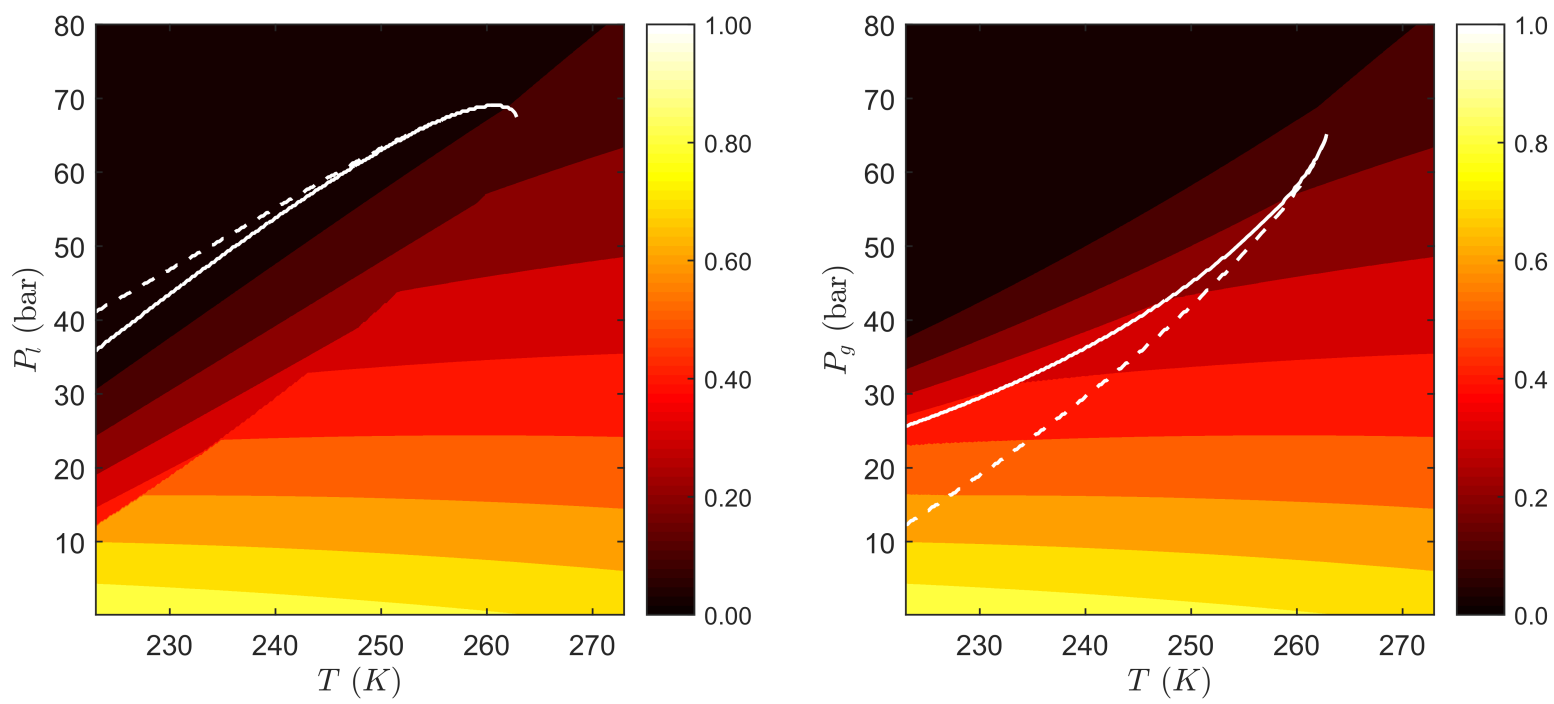

Figure 6: Excess adsorbed phase fraction $(\theta)$ for $\mathrm{C}_{1}-\mathrm{C}_{2}$ binary system. [Left $]$ Liquid phase pressure $P_{l}$. [Right $]$ Gas phase pressure $P_{g}$. Continuous line $(-)$ represents the feed phase pressure, dashed line (- - ) represent the incipient phase pressure. 


\section{Ternary System}

The ternary mixture is constructed in a way to represent a light, intermediate and heavy fraction of an oil mixture. Similar to the binary system, the algorithm shows to be convergent and no particular difficulties were encountered. Figure 7 shows the change of the phase envelope and excess adsorbed phase fraction at different $\mathrm{T}$ and $\mathrm{P}$, and Figure 8 shows the change in the composition of $\mathrm{C}_{10}$ in the bulk phase and the absolute composition in the adsorbed phase.

A shrunk phase envelope due to capillary pressure and compositional changes is observed in Figure 7. This behavior is consistent with the observation in the binary system presented before in Figure 4. In addition, the excess adsorbed phase fraction in the liquid region is close to zero and appears to be less sensitive to changes in the pressure and the temperature than that in the binary mixture. Nevertheless, the composition of decane in the adsorbed phase is very sensitive to changes in the temperature. Therefore, in spite of the apparent zero value of the excess fraction in the liquid region, the individual contributions $n_{i}^{e x c}$ are not negligible in the compositional changes of the bulk phase. The impact of these compositional changes can be observed in the modified cricondentherm of the phase envelope in Figure 4.

If we select an arbitrary temperature in the liquid region and start decreasing the pressure, as soon as the mixture enters the two-phase region, the change of the bulk phase composition $\mathbf{z b}$ becomes become more sensitive to the pressure as shown in Figure 8. In order to better illustrate the composition changes, two fixed reservoir temperatures of $400 \mathrm{~K}$ and $450 \mathrm{~K}$ are selected and the bulk phase compositions $\mathbf{z}^{\mathbf{b}}$ and adsorption selectivities $S_{i, j}{ }^{\mathrm{i}}$ are plotted against the pressure. The plots are presented in Figure 9 and Figure 10 respectively. As the pressure decreases in Figure 9 the bulk phase gets enriched with lighter components due to desorption of $\mathrm{C}_{1}$, while the heavier components remain in the adsorbed phase. As the pressure is further decreased, the adsorption selectivity towards heavier components is enhanced, with dramatic changes when entering the single gas phase region. Both figures

\footnotetext{
${ }^{\mathrm{i}}$ Seletivity of component $i$ with respect to component $j, S_{i, j}=\frac{x_{i}^{a d s} / z_{b_{i}}}{x_{j}^{a d s} / z_{b_{j}}}$
} 
suggest that, in a depletion production scenario, the heavier components will become harder to recover as the pressure of the reservoir decreases. Moreover, they will likely remain in the adsorbed phase at low pressures, making it practically impossible to recover them.
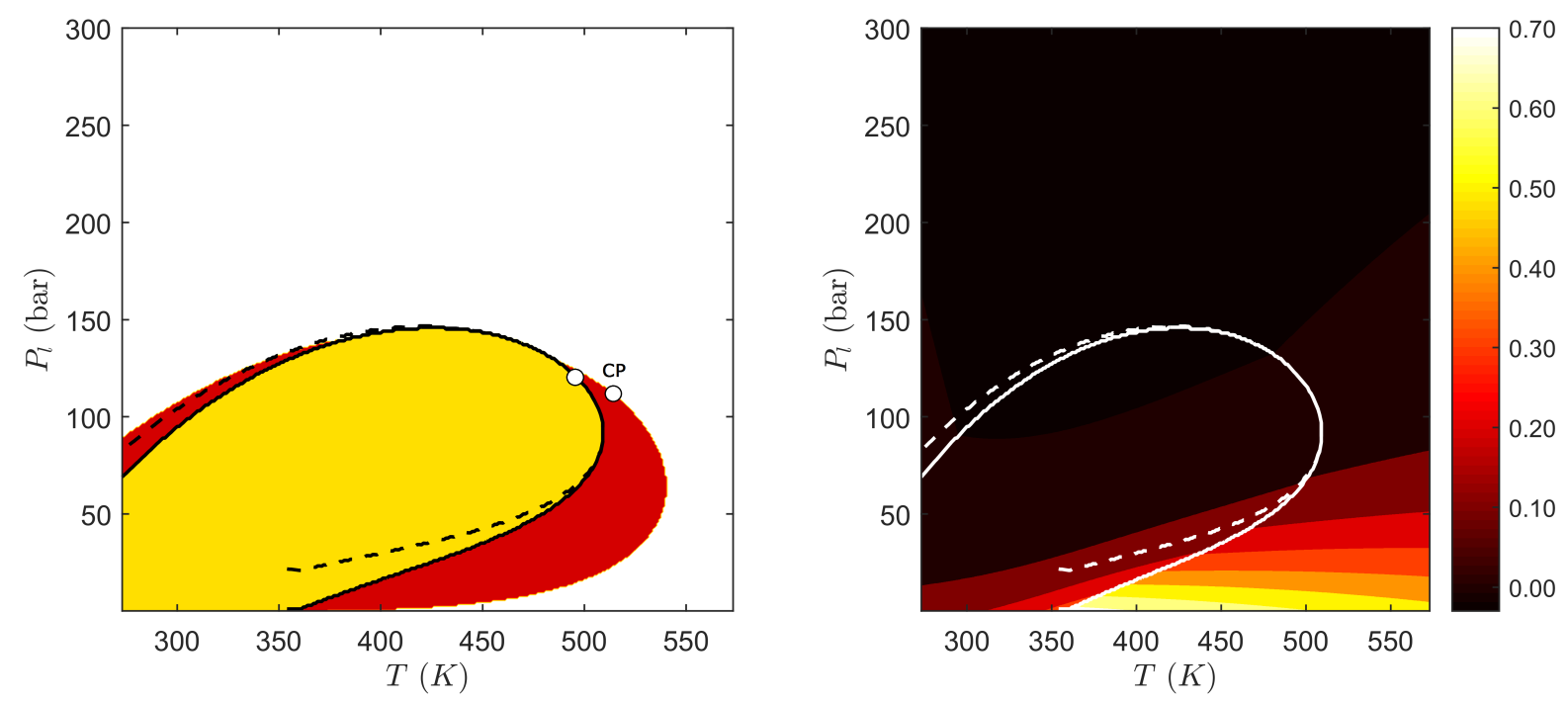

Figure 7: Ternary system. [Left] Normal phase envelope in red; with capillary pressure and adsorption in yellow. [Right] Excess adsorbed phase fraction $(\theta)$ plotted against the liquid phase pressure. Dashed line (- - ) represents the gas phase pressure.
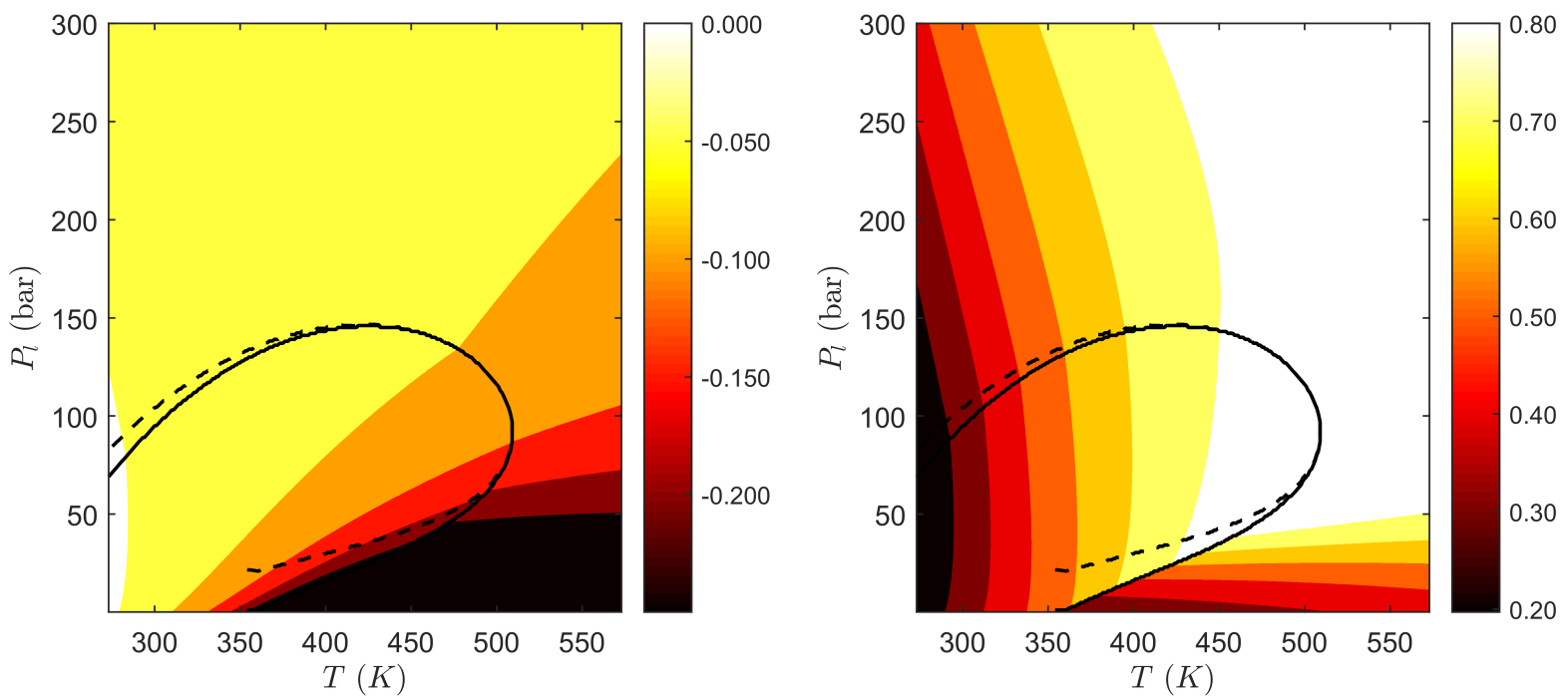

Figure 8: Ternary system. [Left] Change of the $\mathrm{C}_{10}$ mole fraction in the bulk phase $\mathbf{z}_{\mathbf{b}}$. [Right $] \mathrm{C}_{10}$ mole fraction in the absolute adsorbed phase $\mathbf{x}^{\text {abs }}$. 

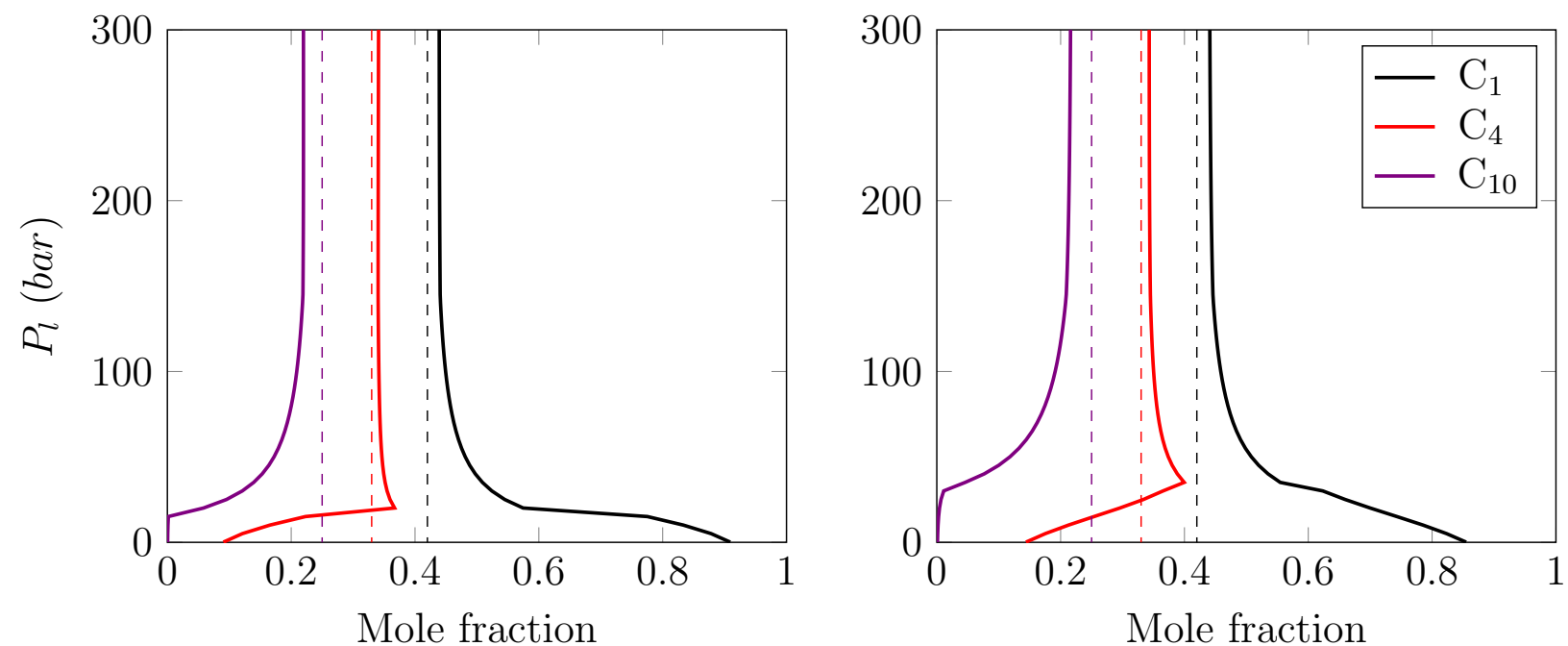

Figure 9: Composition profile of the bulk phase $\left(\mathbf{z}_{\mathbf{b}}\right)$ as a function of the pressure $\left(P_{l}\right)$ at $T=400 K$ and $T=450 K$ respectively.
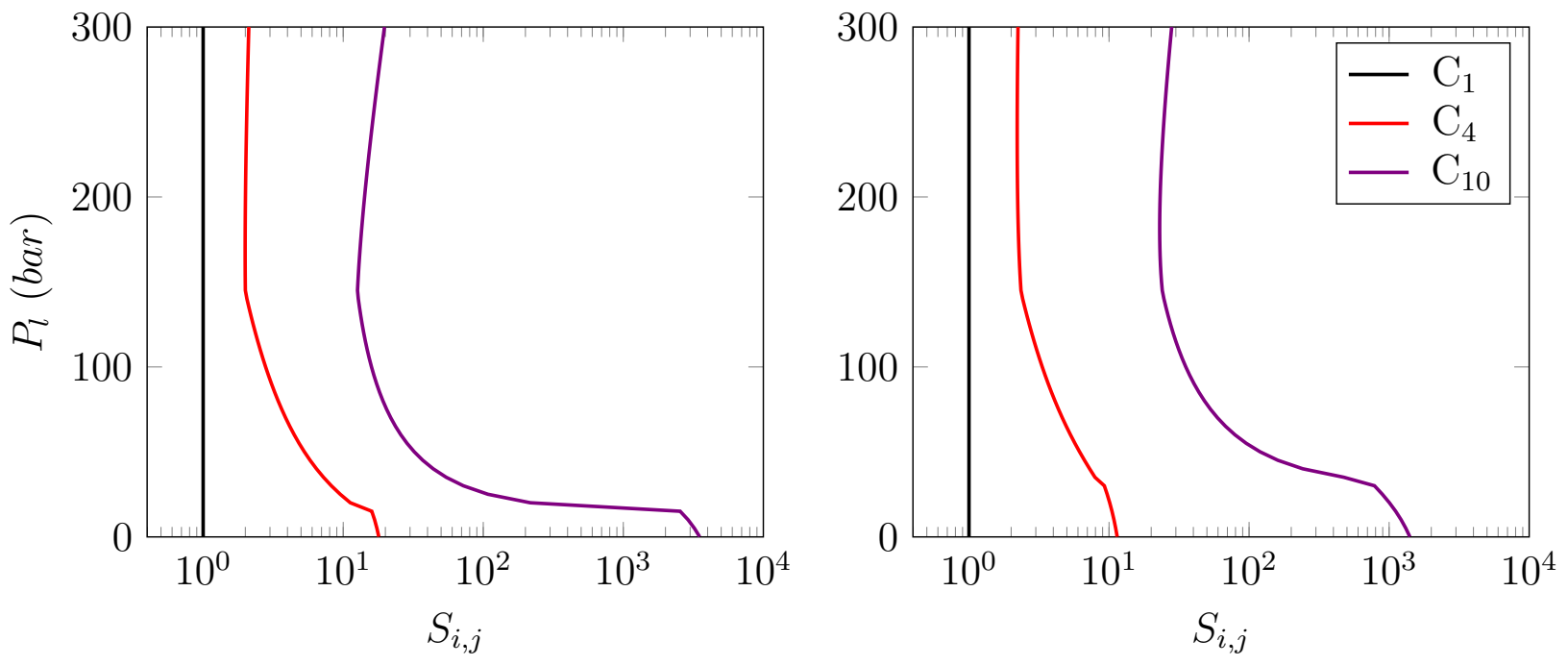

Figure 10: Adsorption selectivity with respect to $\mathrm{C}_{1}$ as a function of the pressure $\left(P_{l}\right)$ at $T=400 K$ and $T=450 K$ respectively. 


\section{Low-GOR and High-GOR Systems}

Two more complex systems that mimic real reservoir fluids are tested. The low-GOR system (lower methane content) represents a volatile oil system and the high-GOR system represents a gas condensate system (higher methane content). The comparison of both are shown from Figure 11 to Figure 14. Once again it can be seen that the two-phase zone shrinks and shifts in the same way as the binary and ternary systems. However, some differences are observed in the high-GOR system. The shift in the upper dew point region is more pronounced in comparison to the other systems. If we fixed a reservoir temperature of $350 \mathrm{~K}$ and decrease the pressure, unlike the other systems, the two-phase region will be reached several bars later than expected as shown in Figure 11. This shows that the shift of the phase envelope due to selective adsorption towards heavier components is more pronounced in some systems than in others. For this particular case, the high-GOR system phase envelope changes more dramatically when small amounts of the heavier hydrocarbons are adsorbed to the wall.

In general, it can be seen that the two-phase zone shrinks when adsorption is considered. However, it is not completely clear how to predict the extent of these changes a priori, since it will depend from system to system. With the considered EoS and adsorption models we can observe that adsorption of heavier alkanes is strongly preferred when there is a gas bulk phase present in the system, this can be seen in the selectivity profiles of Figure 16. In contrast, the preference towards heavier alkanes is low to moderate in the liquid region. For instance, in the liquid region the bulk phase composition $\mathbf{z}^{\mathbf{b}}$ is very similar to that of the overall feed $\mathbf{z}^{\mathbf{f}}$ as shown in the composition profiles of Figure 15. At these temperatures and pressures, the adsorbed phase does not have an obvious effect on the phase equilibrium but it is difficult to predict whether adsorption can be neglected or not. In general, it should be noted that the role of the adsorbed phase will become more relevant when the system changes from a liquid phase zone to a two-phase zone. Therefore, it is recommended not to disregard its effect when the phase equilibrium of a confined system is evaluated. 

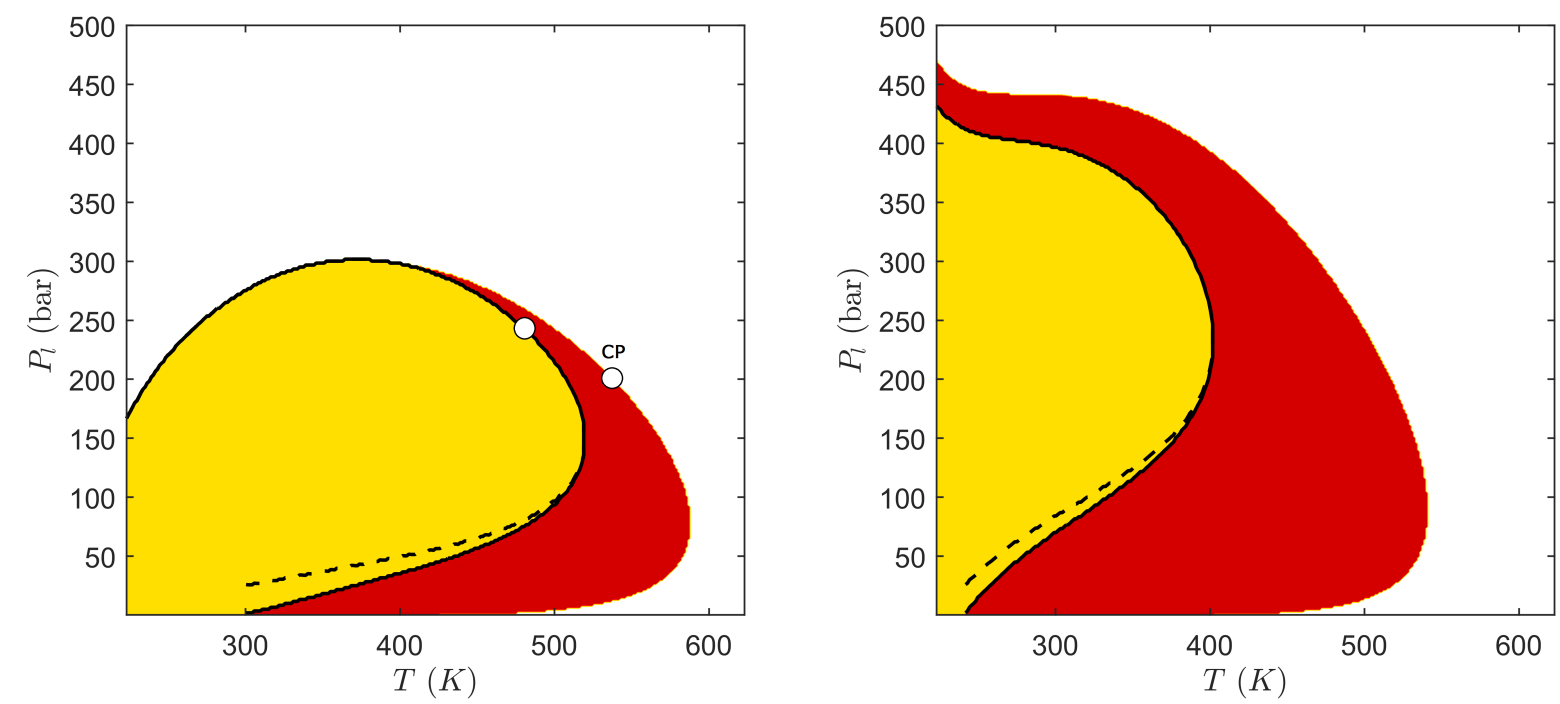

Figure 11: Normal phase envelope in red; with capillary pressure and adsorption in yellow. [Left] Low-GOR. [Right] High-GOR. (No critical point is present in the pressure and temperature range)
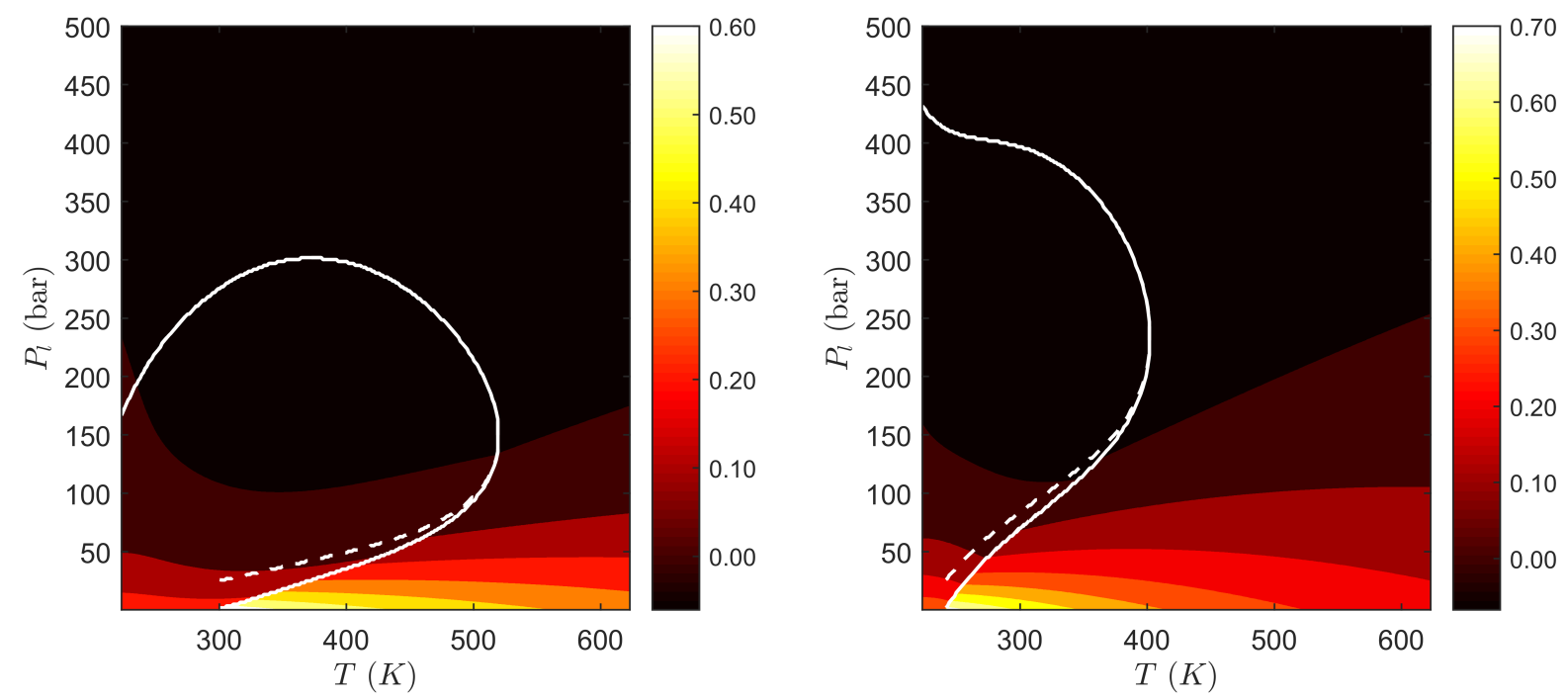

Figure 12: Excess adsorbed phase fraction $(\theta)$ plotted against the liquid phase pressure. Dashed line (- - ) represents the gas phase pressure. [Left] Low-GOR. [Right] High-GOR. 

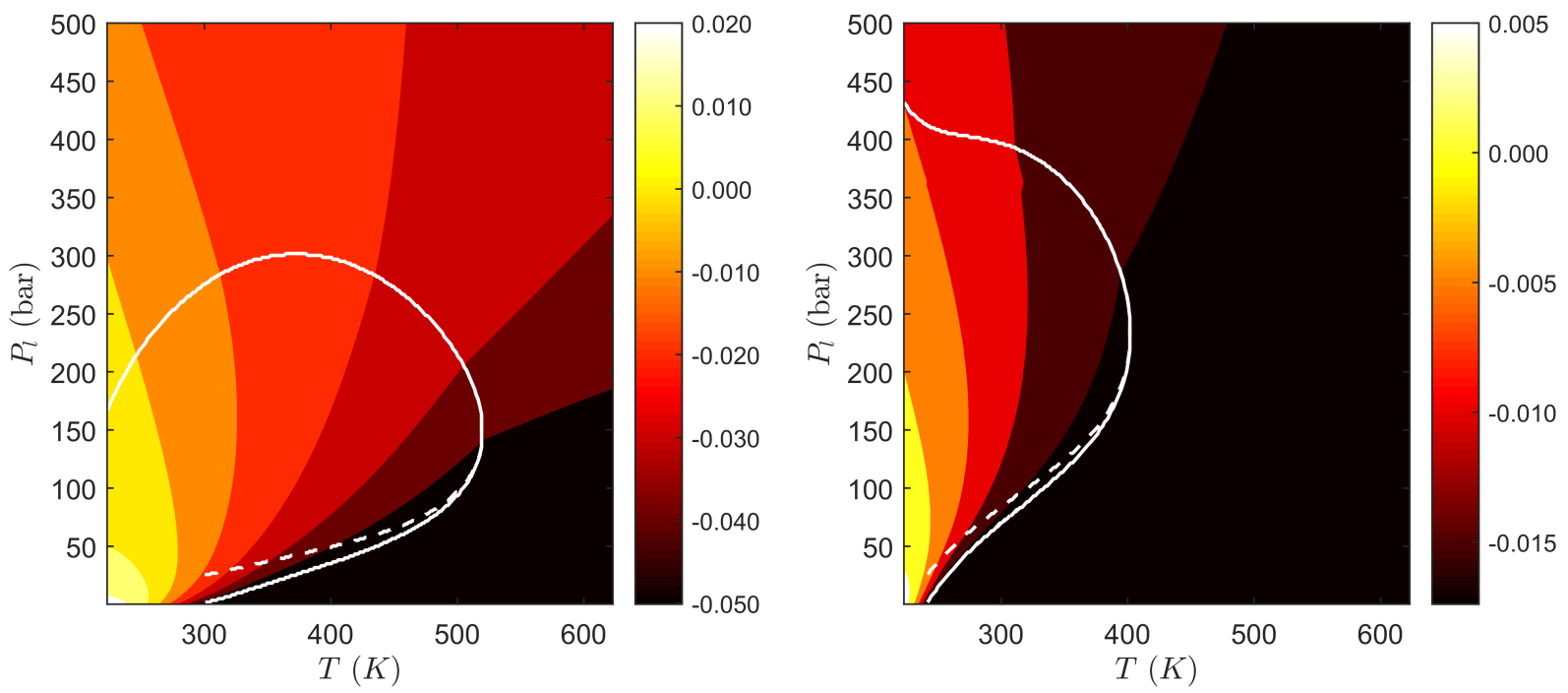

Figure 13: $\mathrm{C}_{16}$ mole fraction change in bulk phase $\mathbf{z}_{\mathbf{b}}$. [Left $]$ Low-GOR, reference feed $\left(z_{C_{16}}=0.501\right)$. [Right] High-GOR, reference feed $\left(z_{C_{16}}=0.0174\right)$.
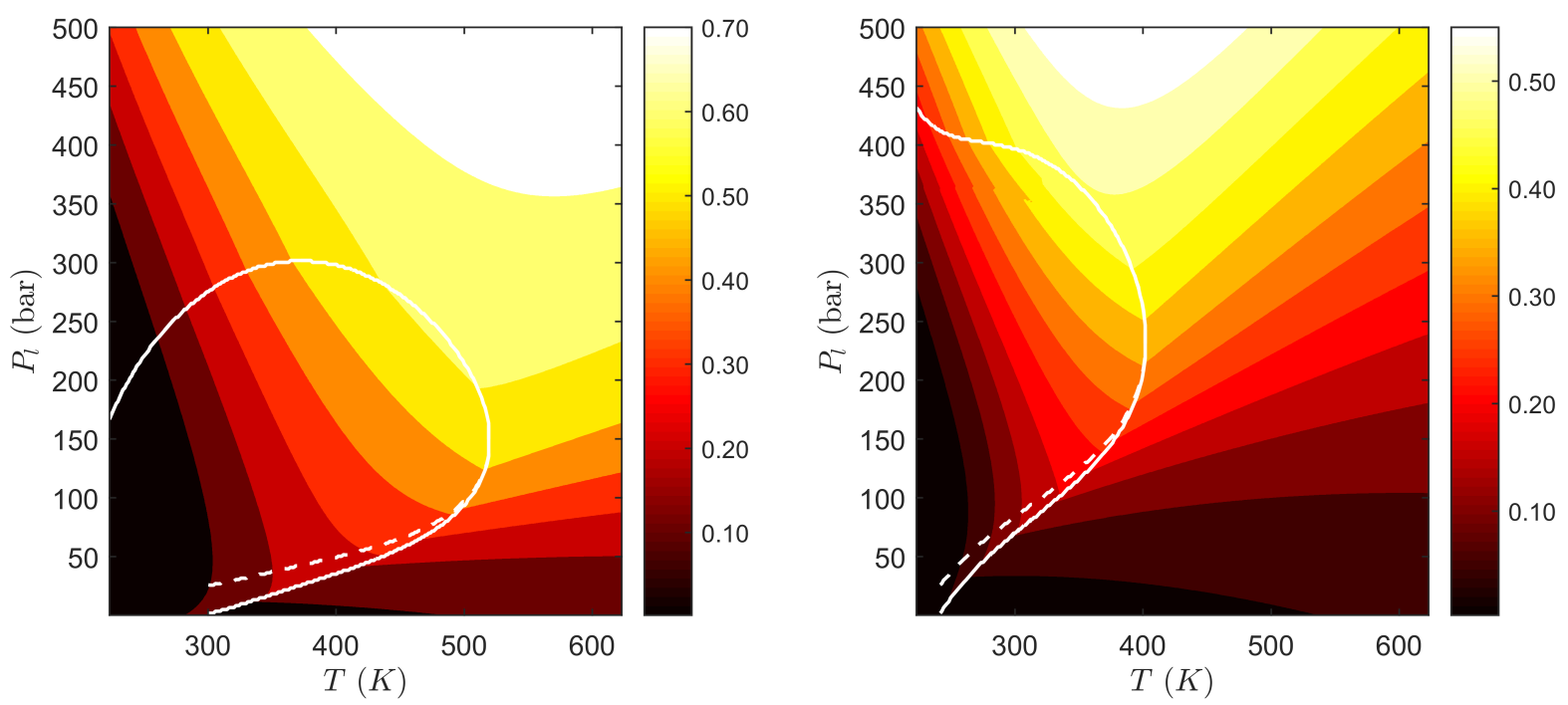

Figure 14: $\mathrm{C}_{16}$ mole fraction in the absolute adsorbed phase $\mathbf{x}^{\mathbf{a b s}}$. [Left $]$ Low-GOR. [Right] High-GOR. 

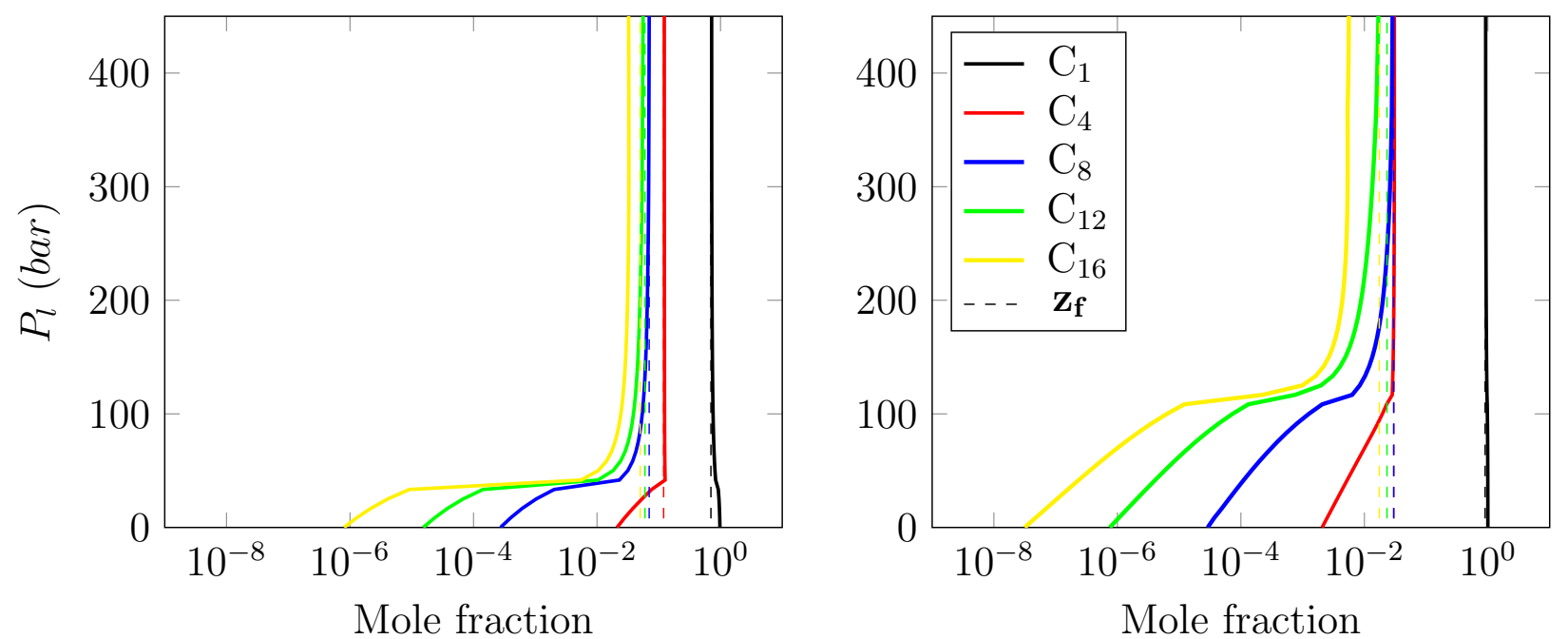

Figure 15: Composition profile of the bulk phase $\left(\mathbf{z}_{\mathbf{b}}\right)$ as a function of the pressure $\left(P_{l}\right)$. $[$ Left $]$ Low-GOR at $T=400 \mathrm{~K}$. [Right $]$ High-GOR at $T=350 \mathrm{~K}$.
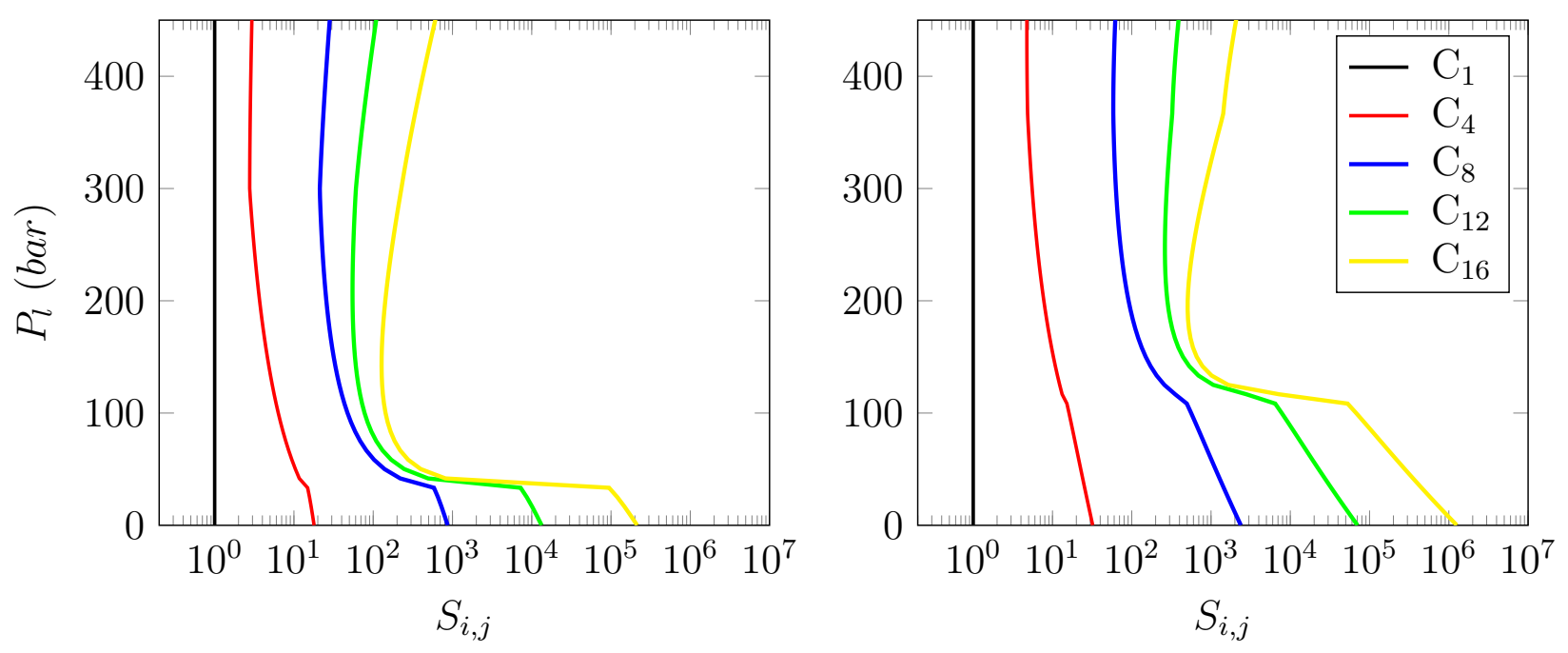

Figure 16: Adsorption selectivity with respect to $\mathrm{C}_{1}$ as a function of the pressure $\left(P_{l}\right)$. [Left $]$ Low-GOR at $T=400 \mathrm{~K}$. [Right $]$ High-GOR at $T=350 \mathrm{~K}$. 
The above analysis for the binary, ternary, and multicomponent systems has revealed the shifts of phase envelopes under the combined effects of capillary pressure and adsorption. Ideally, such shifts should be validated quantitatively and qualitatively by experimental data. However, to the best of our knowledge, there are no experimental results which can be used for such purpose. Among the recent experimental studies ${ }^{8-13}$ briefly mentioned in the introduction section, most of them investigate just the capillary pressure effects ${ }^{8,9}$ or at least do not explicitly include the adsorption effects $\left.{ }^{10-13}\right)$. Hence, a direct comparison with the calculation here cannot be made.

In the studies using nanofluidics, ${ }^{8,9}$ vaporization tends to happen first in the bigger channels used as the inlet and outlet, making it difficult to interpret the results for mixtures ${ }^{8}$ due to diffusion of components across the system. Alfi et al. ${ }^{9}$ studied only pure components and the results are qualitatively in agreement with the bubble point suppression predicted by the Kelvin equation. Furthermore, the results of Luo et al. ${ }^{11-13}$ show two-phase transition points: one above the original saturation temperature and one below. This phenomenon was not observed in the experiments by the others. ${ }^{8-10}$ It should also be noted that most of these experiments are at atmospheric conditions ${ }^{8,9,11-13}$ which are far from the high pressure underground conditions of interest to us and analyzed in this study. Furthermore, Pathak et al. ${ }^{10}$ reported a bubble point pressure suppression for methane-n-decane in porous media at elevated pressures. Their bubble point pressures were determined from the pressure-volume curve. However, we notice that their reported curves are very smooth and the determined bubble point pressures can be subject to large uncertainty, therefore, difficult for a direct quantitative comparison.

In general, it is challenging to measure saturation point inside porous media because no stirring can be made to ensure the homogeneity of the fluid system. Despite the deficiencies in the existing experimental studies, they are still valuable attempts and will pave the way for improved studies in the future. It is just at the current stage that a quantitative comparison with our calculation for high pressure mixtures is not possible. Finally, it should be noted 
that the quantitative results depend on the system and the adsorption model used. We tried to develop our adsorption modeling based on the experimental adsorption data on a particular shale sample. ${ }^{26}$ Therefore, the actual extent of the phase behavior shift will vary with the adsorbent.

\section{PVT Analysis}

The phase envelope calculation provides an overall picture of the phase behavior in the reservoir. For shale producers, direct PVT analyses are more compelling since they simulate a path close to a real production scenario during depletion. To give a better insight on the influence of the capillary pressure and adsorption, we performed PVT simulations for both the low-GOR (volatile oil) and high GOR (gas condensate) systems. We simulated Constant Mass Expansion (CME) for both systems, and Differential Liberation (DL) for the low-GOR system and Constant Volume Depletion (CVD) for the high-GOR system. In order to do the simulations with capillary pressure and adsorption, the classical two-phase flash used in PVT simulations were replaced by the flash with capillary pressure and adsorption developed in this work. For CME, the simulation is straightforward since the mass is conserved. For DL, the gas phase formed at a pressure stage below the bubble point pressure is removed completely, leaving only the adsorbed and liquid phases before proceeding to the next pressure stage. For CVD, the system volume is kept at the dew point volume for pressure stages below the dew point pressure by removing the excess amount of gas. For Low-GOR, we simulated CME and DL at $400 \mathrm{~K}$ and for high-GOR, we simulated CME and CVD at $350 \mathrm{~K}$. The two temperatures were selected arbitrarily within the normal range of temperatures for shale reservoirs.

\section{Low-GOR fluid}

The PVT calculation results are depicted in Figure 19. It can be observed that when capillary pressure and adsorption are considered, the results deviate significantly from the normal 
or conventional PVT results. Figure 19a shows that the oil formation volume factor $B_{o}$ is larger in all the pressure range when considering capillarity and adsorption for both the CME and DL tests. This difference gets smaller for lower pressures in the two-phase region. A larger $B_{o}$ means a lighter oil phase is obtained. This is mostly attributed to the adsorption of heavier components to the rock since the capillary pressure plays a minor role as indicated by the nearly unchanged bubble point pressure. The gas formation volume factor $B_{g}$ shown in Figure 19b shows a very small difference between the CME and DL tests, and also small differences at high pressures when considering capillary and adsorption. At lower pressures the influence of adsorption in $B_{g}$ becomes more noticeable, which is consistent with the results obtained in the corresponding phase envelope. As the system enters a gas rich region, the influence of adsorption in the system increases. Figure 19c presents the solution Gas-Oil ratio $R_{s}$. Similar to the $B_{o}$, the $R_{s}$ is larger than the normal case in all the pressure range when considering capillarity and adsorption. As the pressure decreases, this difference gets smaller. In contrast to the normal $R_{s}$ plot, when considering capillarity and adsorption the $R_{s}$ is not constant before the bubble pressure is reached. Since the adsorbed phase changes slightly above the bubble point pressure, the bulk composition $z_{b}$ changes as well resulting in small changes of $R_{s}$. Finally, a liquid density plot $\rho_{l}$ is presented in Figure 19d. As can be expected from the $B_{o}$ plots, the density of the liquid phase decreases for the results with capillary pressure and adsorption. This difference exists in the whole pressure range and get smaller at lower pressures.

\section{High-GOR fluid}

The results are shown in Figure 20. Similarly, the high-GOR system also shows changes in the PVT results when including capillary pressure and adsorption. Figure 20a shows the solution oil-gas ratio $r_{s}$ of the produced stream as function of the pressure. We can again observe that the $r_{s}$ slightly varies at pressures higher than the bubble point due to compositional changes caused by desorption. When considering capillarity and adsorption, the $r_{s}$ 
stays almost constant after the normal dew point (ca. 428 bar) for about 60 bar until the modified dew point is met (ca. 365 bar). The results seem to be in qualitative agreement with the field observations that the produced gas-oil ratio remains constant even after the apparent saturation point is passed. Furthermore, the $r_{s}$ with capillary pressure and adsorption seems to decrease at a slower rate than the normal system after the dew point. This may be partly attributed to the release of heavy components from the adsorbed phase to the bulk phase favouring the condensation at standard conditions. Figure 20b shows the gas formation volume factor with almost no difference in the whole pressure range because the density change in the gas phase due to adsorption is limited. Figure 20c shows the liquid dropout $V_{r l}$ (liquid volume divided by the saturation point volume) for the different tests. For the normal CME and CVD case the liquid dropout is considerably higher. Because of the shifted dew point, the condensation inside the PVT cell for the normal case starts earlier with a very steep increasing change in $V_{r l}$ and then decreases slowly as the pressure is lowered. For the results with capillary pressure and adsorption, the first liquid drop appears around 60 bars below the normal dew point. The change in $V_{r l}$ is not as dramatic as for the normal case. For the CME case, the $V_{r l}$ vanishes after entering the gas phase zone (ca. 110 bar) where all the liquid phase re-vaporizes. For the CVD case the $V_{r l}$ reaches almost a constant value due to the enrichment of the bulk phase with heavier components desorbed from the wall after each gas discharge at every stage. Finally, a plot of the $y_{7+}$ mole fraction of the produced stream is shown in Figure 20d. The plot shows a trend similar to that for $r_{s}$ because the condensate at standard conditions includes the majority of the heavy components; the concentration in the gas stream remains almost constant after the normal dew point is reached and starts decreasing after the shifted dew point is reached.

The above PVT analysis is only for two specific model reservoir fluid systems. It is worth pointing out that the PVT simulation results under the capillary pressure and adsorption effects can be influenced by many factors such as the reservoir fluid type, the fluid compo- 
sition, the pore sizes, the adsorption curves, and the reservoir temperatures. More detailed analysis can be made in the future with the flash calculation tool developed here. It should also be pointed out that the perceived in-situ composition of a shale reservoir is crucial for any discussion about the phase equilibrium change in that reservoir. In our discussion here, it was assumed that the overall composition including the adsorbed phase is known. In practice, there is still no consensus on how to get representative samples from shale reservoirs. ${ }^{44}$ One potential route is to combine reservoir simulation with PVT analysis. ${ }^{45}$ For that purpose, the flash calculation framework developed here can be integrated into a compositional simulator to include both capillary pressure and adsorption effects. Such a simulator will allow dynamic analysis more relevant to actual shale production. 

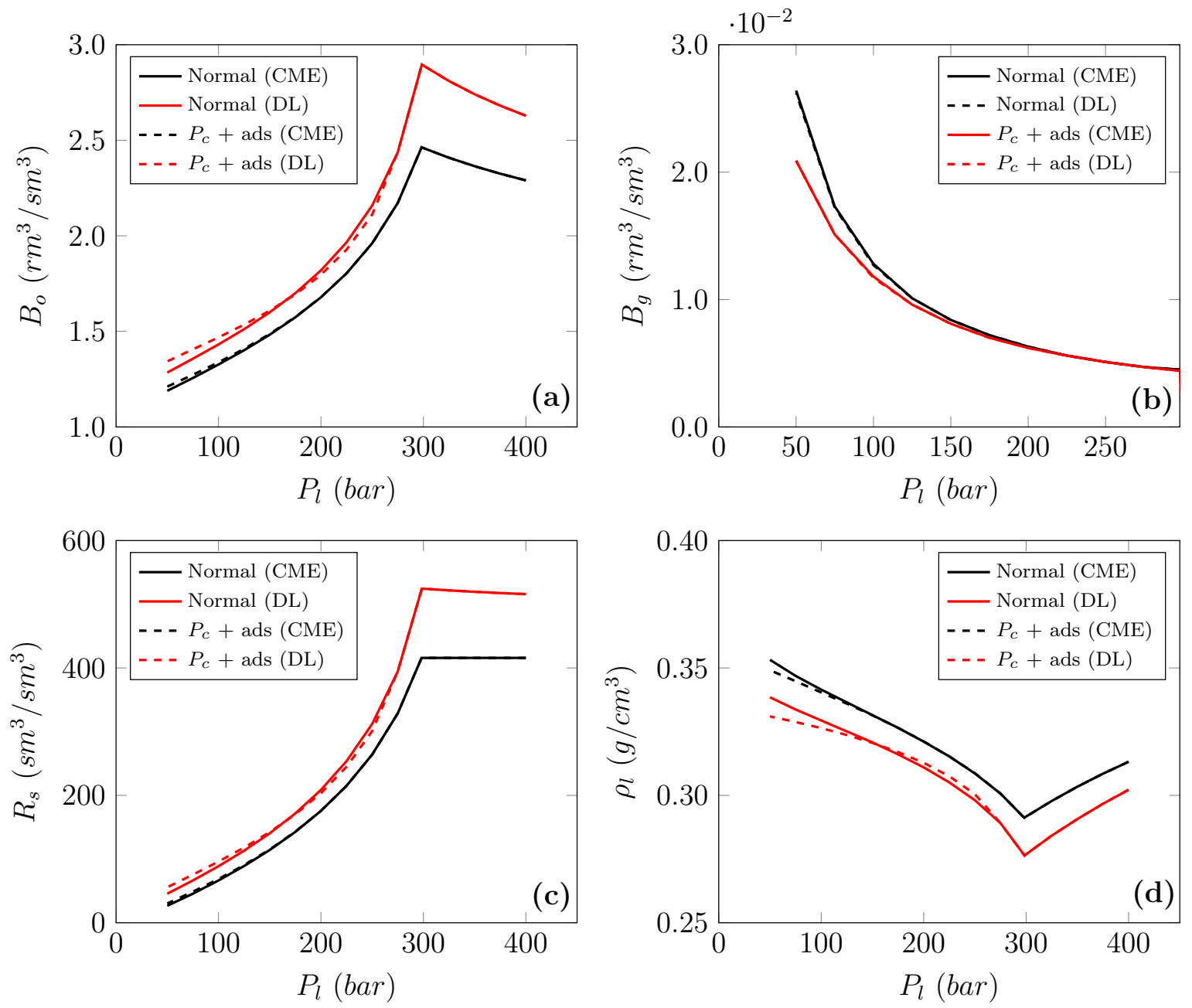

Figure 17: (a) Oil formation volume factor $B_{o}$, (b) Gas formation volume factor $B_{g}$, (c) Solution gas-oil ratio $R_{s}$, and (d) liquid density $\rho_{l}$ for $\mathrm{CME}$ and DL tests at $\mathrm{T}=400 \mathrm{~K}$ using the low-GOR fluid. 

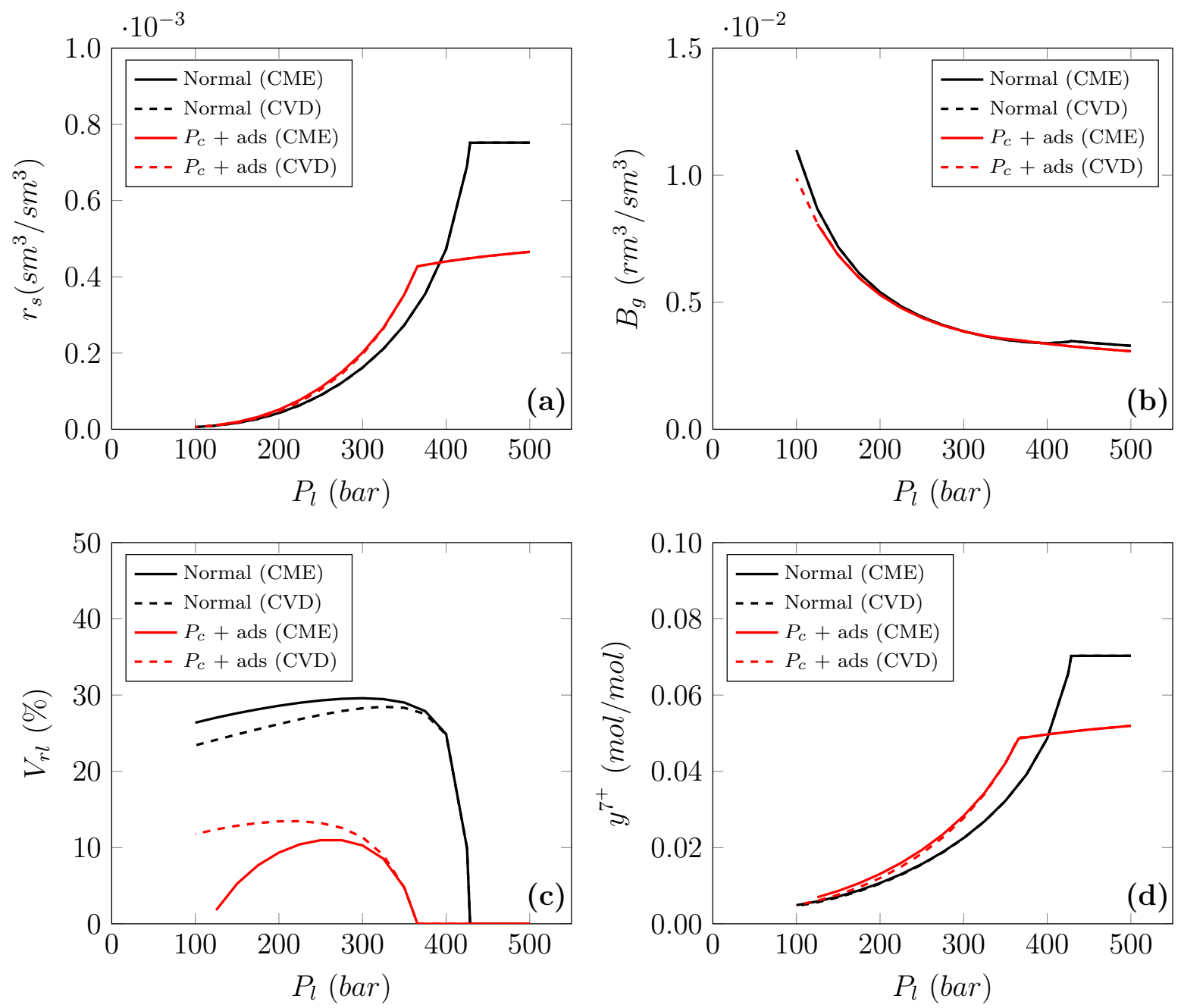

Figure 18: (a) Solution oil-gas ratio $r_{s}$, (b) Gas formation volume factor $B_{g}$, (c) liquid drop out $V_{r l}$, and (d) $y^{7}+$ mole fraction in the producing stream for CME and CVD tests at $\mathrm{T}=400 \mathrm{~K}$ using the high-GOR fluid. 


\section{Conclusions}

In this work, the combined effects of capillarity and adsorption were investigated. A flash algorithm including both capillary pressure and adsorption was developed to determine the phase fractions and compositions of two bulk phases (liquid and gas) and an adsorbed phase at equilibrium. The algorithm is based on a two-phase flash procedure that accounts for the capillary pressure difference between the liquid and gas phases, and the compositional changes in the bulk caused by selective adsorption of components to the wall.

The flash algorithm was used to analyze some representative systems (binary, ternary, low GOR, and high GOR model systems) for the phase equilibrium in shale. The results show that adsorption and capillary pressure can significantly change the bulk phase composition and thus its corresponding phase envelope. Since the adsorption is different at different temperature and pressure conditions, the extent of change is different. In general, a much shrunk phase envelope with a shifted critical point is observed. The effect of capillary pressure shows to be more important close to the bubble point boundary, where the adsorption effects are often moderate. The adsorption selectivity towards heavier components at high pressure and low temperature liquid region is from low to moderate, and the excess adsorbed phase fraction in the liquid phase region is close to zero. On the other hand, the adsorption of heavier alkanes is strongly preferred in the low pressure and high temperature gas phase region. Moreover, the excess adsorbed phase fraction in the gas phase region can be as high as $80 \%$. The high selectivity towards heavier alkanes and the large excess adsorbed phase fraction in the gas phase region can lead to dramatic changes in the bulk composition. Consequently, a big shift is observed in the phase envelope along the dew point branch.

In addition, PVT analysis using the developed flash algorithm with adsorption and capillary pressure was made for the low-GOR and high-GOR fluid, revealing significant shifts from the conventional PVT results. For the low-GOR fluid/volatile oil, CME and DL tests were simulated. The solution gas-oil ratio and the oil formation volume factor increases in the entire pressure range under the influence of adsorption and capillary pressure. For the 
hig-GOR fluid, CME and CVD tests were simulated. Because of the shifted upper dew point pressure, a relatively constant segment below the original dew point pressure is observed in the solution oil-gas ratio and the $y_{7+}$ mole fraction of the produced stream for the simulation with capillary pressure and adsorption. In general, the results will strongly depend on the choice of the reservoir fluid and temperature. The extent of the influence of the capillary pressure and adsorption may vary significantly as can be observed on their phase envelopes.

\section{Supporting Information}

The Supporting Information is available free of charge on the ACS Publication website.

- Artificial Adsorption Data Generated with MPTA

\section{Acknowledgments}

We would like to acknowledge ConocoPhillips and ExxonMobil for their financial support. We are grateful to Prof. Alexander Shapiro for the valuable discussions.

\section{References}

(1) Alexander, T.; Baihly, J.; Boyer, C.; Clark, B.; Waters, G.; Jochen, V.; Le Calvez, J.; Lewis, R.; Miller, C. K.; Thaeler, J.; Toelle, B. E. Shale Gas Revolution. Oilfield Review 2011, 23, 40-55.

(2) Santos, V. E. S.; Rego, E. E.; dos Santos, E. M.; Ribeiro, C. O. Shale Gas and the Replacement of Coal-Fired Power Plants. IEEE Latin America Transactions 2016, 14, $3721-3730$.

(3) Faouzi Aloulou, V. Z. International Energy Outlook 2016; , 2016. 
(4) Clarkson, C.; Pedersen, P. Production Analysis of Western Canadian Unconventional Light Oil Plays (SPE-149005-MS). SPE Canadian Unconventional Resources Conference. Calgary, Alberta, Canada, 2011.

(5) Nojabaei, B.; Johns, R. T.; Chu, L. Effect of Capillary Pressure on Phase Behavior in Tight Rocks and Shales. SPE Reservoir Eval. Eng. 2013, August, 281-289.

(6) Kurtoglu, B.; Kazemi, H. Evaluation of Bakken performance using coreflooding, well testing, and reservoir simulation (SPE-155655-MS). SPE Annual Technical Conference and Exhibition. San Antonio, Texas, USA, 2012.

(7) Fisher, L.; Israelachvili, J. Experimental studies on the applicability of the Kelvin equation to highly curved concave menisci. J. Colloid Interface Sci. 1981, 80, 528-541.

(8) Wang, L.; Parsa, E.; Gao, Y.; Ok, J. T. Experimental Study and Modeling of the Effect of Nanoconfinement on Hydrocarbon Phase Behavior in Unconventional Reservoirs (SPE-169581-MS). SPE Western North American and Rocky Mountain Joint Meeting. Denver, Colorado, USA, 2014.

(9) Alfi, M.; Nasrabadi, H.; Banerjee, D. Experimental investigation of confinement effect on phase behavior of hexane, heptane and octane using lab-on-a-chip technology. Fluid Phase Equilib. 2016, 423, 25 - 33.

(10) Pathak, M.; Cho, H.; Deo, M. Experimental and Molecular Modeling Study of Bubble Points of Hydrocarbon Mixtures in Nanoporous Media. Energy Fuels 2017, 31, 34273435.

(11) Luo, S.; Lutkenhaus, J. L.; Nasrabadi, H. Confinement-Induced Supercriticality and Phase Equilibria of Hydrocarbons in Nanopores. Langmuir 2016, 32, 11506-11513.

(12) Luo, S.; Nasrabadi, H.; Lutkenhaus, J. L. Effect of Confinement on the Bubble Points of Hydrocarbons in Nanoporous Media. AIChE J. 2016, 62, 1772-1780. 
(13) Luo, S.; Lutkenhaus, J. L.; Nasrabadi, H. Use of differential scanning calorimetry to study phase behavior of hydrocarbon mixtures in nano-scale porous media. J. Pet. Sci. Eng. 2016, 1-8.

(14) Brusilovsky, A. I. Mathematical Simulation of Phase Behavior of Natural Multicomponent Systems at High Pressures With an Equation of State. SPE 1992, February, $117-122$.

(15) Shapiro, A.; Stenby, E. Kelvin equation for a non-ideal multicomponent mixture. Fluid Phase Equilib. 1997, 134, 87-101.

(16) Shapiro, A.; Stenby, E. Thermodynamics of the multicomponent vapor liquid equilibrium under capillary pressure difference. Fluid Phase Equilib. 2001, 178, 17-32.

(17) Shapiro, A.; Stenby, E. Effects of Capillary Forces and Adsorption on Reserves Distribution (SPE 36922). SPE European Petroleum Conference. Milan, Italy, 1996; pp $441-448$.

(18) Sandoval Lemus, D. R.; Yan, W.; Michelsen, M. L.; Stenby, E. H. The Phase Envelope of Multicomponent Mixtures in the Presence of a Capillary Pressure Difference. Ind. Eng. Chem. Res. 2016, 55, 6530-6538.

(19) Pang, J.; Zuo, J.; Zhang, D.; Du, L.; Corporation, H. Effect of Porous Media on Saturation Pressures of Shale Gas and Shale Oil (IPTC 16419). International Petroleum Technology Conference. Beijing, China, 2013; pp 1-7.

(20) Firincioglu, T.; Llc, N.; Ozgen, C.; Ozkan, E. An Excess-Bubble-Point-Suppression Correlation for Black Oil Simulation of Nano-Porous Unconventional Oil Reservoirs (SPE 166459) . SPE Annual Technical Conference and Exhibition. New Orleans, Louisiana, USA, 2013. 
(21) Dong, X.; Liu, H.; Hou, J.; Wu, K.; Chen, Z. Phase Equilibria of Confined Fluids in Nanopores of Tight and Shale Rocks Considering the Effect of Capillary Pressure and Adsorption Film. Ind. Eng. Chem. Res. 2016, 55, 798-811.

(22) Gasparik, M.; Ghanizadeh, A.; Bertier, P.; Gensterblum, Y.; Bouw, S.; Krooss, B. M. High-Pressure Methane Sorption Isotherms of Black Shales from the Netherlands. Energy Fuels 2012, 26, 4995-5004.

(23) Gasparik, M. et al. First international inter-laboratory comparison of high-pressure $\mathrm{CH} 4, \mathrm{CO} 2$ and $\mathrm{C} 2 \mathrm{H} 6$ sorption isotherms on carbonaceous shales. Int. J. Coal Geol. 2014, 132, 131-146.

(24) Luo, X.; Wang, S.; Wang, Z.; Jing, Z.; Lv, M.; Zhai, Z.; Han, T. Adsorption of methane, carbon dioxide and their binary mixtures on Jurassic shale from the Qaidam Basin in China. Int. J. Coal Geol. 2015, 150-151, 210-223.

(25) Rexer, T. F.; Mathia, E. J.; Aplin, A. C.; Thomas, K. M. High-Pressure Methane Adsorption and Characterization of Pores in Posidonia Shales and isolated kerogens. Energy Fuels 2014, 28, 2886-2901.

(26) Wang, Y.; Tsotsis, T. T.; Jessen, K. Competitive Sorption of Methane/Ethane Mixtures on Shale: Measurements and Modeling. Ind. Eng. Chem. Res. 2015, 54, 12187-12195.

(27) Zhang, T.; Ellis, G. S.; Ruppel, S. C.; Milliken, K.; Yang, R. Effect of OrganicMatter Type and Thermal Maturity on Methane Adsorption in Shale-Gas Systems. Org. Geochem. 2012, 47, 120-131.

(28) Collell, J.; Galliero, G.; Gouth, F.; Montel, F.; Pujol, M.; Ungerer, P.; Yiannourakou, M. Molecular Simulation and Modelisation of Methane/Ethane Mixtures Adsorption onto a Microporous Molecular Model of Kerogen under Typical Reservoir Conditions. Microporous Mesoporous Mater. 2014, 197, 194-203. 
(29) Zhang, H.; Zeng, X.; Zhao, Z.; Zhai, Z.; Cao, D. Adsorption and selectivity of CH4/CO2 in functional group rich organic shales. J. Nat. Gas Sci. Eng. 2017, 39, $82-89$.

(30) Mason, J. A.; Veenstra, M.; Long, J. R. Evaluating metal-organic frameworks for natural gas storage. Chem. Sci. 2014, 5, 32-51.

(31) Sandoval, D. R.; Yan, W.; Michelsen, M. L.; Stenby, E. H. Modeling of Shale Gas Adsorption and Its Influence on Phase Equilibrium. Ind. Eng. Chem. Res. 2017, (Web).

(32) Leverett, M.; Lewis, W.; True, M. Dimensional-model Studies of Oil-field Behavior. SPE J. 1942,

(33) Chenevert, M.; Osisanya, O. Shale swelling at elevated temperature and pressure (ARMA-92-0869). The 33th U.S. Symposium on Rock Mechanics. Santa Fe, New Mexico, USA, 1992.

(34) Lu, Y.; Ao, X.; Tang, J.; Jia, Y.; Zhang, X.; Chen, Y. Swelling of shale in supercritical carbon dioxide. J. Nat. Gas Sci. Eng. 2016, 30, 268-275.

(35) Lyu, Q.; Ranjith, P. G.; Long, X.; Kang, Y.; Huang, M. A review of shale swelling by water adsorption. J. Nat. Gas Sci. Eng. 2015, 27, 1421-1431.

(36) Michelsen, M.; Mollerup, J. Thermodynamic models; Fundamentals and Computational aspects; 1998.

(37) Orbach, O.; Crowe, C. M. Convergence promotion in the simulation of chemical processes with recycle the dominant eigenvalue method. Can. J. Chem. Eng. 1971, 49, 509-13, 509-513.

(38) Sherafati, M.; Jessen, K. Stability analysis for multicomponent mixtures including capillary pressure. Fluid Phase Equilib. 2017, 433, 56-66.

(39) Myers, A. L.; Prausnitz, J. M. Thermodynamics of Mixed-Gas Adsorption. AIChE J. $1965,11,121-127$. 
(40) Shapiro, A.; Stenby, E. Potential Theory of Multicomponent Adsorption. J. Colloid Interface Sci. 1998, 157, 146-157.

(41) Collell, J.; Galliero, G. Theoretically Based Model for Competitive Adsorption of Subcritical Mixtures. J. Phys. Chem. C 2014, 118, 26162-26171.

(42) Sandoval, D. R. Phase Equilibrium Modeling for Shale Production Simulation. Ph.D. thesis, Technical University of Denmark, Lyngby, Denmark, 2017.

(43) Schechter, D. S.; Guo, B. Parachors Based on Modern Physics and Their Uses in IFT Prediction of Reservoir Fluids. SPE J. 1998, June, 207-217.

(44) Honarpour, M. M.; Nagarajan, N. R.; Orangi, A.; Arasteh, F.; Yao, Z. Characterization of Critical Fluid, Rock, and Rock-Fluid Properties-Impact on Reservoir Performance of Liquid-rich Shales (SPE 158042). SPE Annual Technical Conference and Exhibition. San Antonio, Texas, USA, 2012; pp 1-22.

(45) Whitson, C. H.; Sunjerga, S. PVT in Liquid-Rich Shale Reservoirs (SPE 155499). SPE Annual Technical Conference and Exhibition. San Antonio, Texas, USA, 2012; pp 8-10. 